SISSA 105/2003/FM

\title{
Integrable field theory and critical phenomena. The Ising model in a magnetic field
}

\author{
Gesualdo Delfino \\ International School for Advanced Studies (SISSA) \\ via Beirut 2-4, 34014 Trieste, Italy \\ INFN sezione di Trieste \\ E-mail: delfino@sissa.it
}

\begin{abstract}
The two-dimensional Ising model is the simplest model of statistical mechanics exhibiting a second order phase transition. While in absence of magnetic field it is known to be solvable on the lattice since Onsager's work of the forties, exact results for the magnetic case have been missing until the late eighties, when A. Zamolodchikov solved the model in a field at the critical temperature, directly in the scaling limit, within the framework of integrable quantum field theory. In this article we review this field theoretical approach to the Ising universality class, with particular attention to the results obtained starting from Zamolodchikov's scattering solution and to their comparison with the numerical estimates on the lattice. The topics discussed include scattering theory, form factors, correlation functions, universal amplitude ratios and perturbations around integrable directions. Although we restrict our discussion to the Ising model, the emphasis is on the general methods of integrable quantum field theory which can be used in the study of all universality classes of critical behaviour in two dimensions.
\end{abstract}




\section{Introduction}

A lattice system close to a second order phase transition point exhibits a number of features which do not depend on the specific microscopic realisation and coincide with those of all systems sharing the same essential symmetry properties in the given spatial dimension. In principle, the continous field theoretical description of the scaling region provides the most natural theoretical framework for the quantitative study of these universal features; in practice, however, the need of non-perturbative methods seriously complicates the task.

The Ising model [1] is the fundamental model in the theory of critical phenomena. Its theoretical importance became evident in 1944, when Onsager was able to compute the free energy on the square lattice in absence of magnetic field, providing in this way the first exact description of a second order phase transition [2]. It had to become clear later that the Ising model corresponds to the simplest universality class of critical behaviour. Since Onsager's work, the Ising model has been an essential indicator of the progress in the analytic study of critical phenomena. While it remains unsolved in three dimensions, the lattice studies gave additional exact results in the two dimensional case. In 1952 Yang published the first derivation of the formula for the spontaneous magnetisation that Onsager had presented three years before [3]. Scaling theory would later show that this result amounted to completing the list of critical exponents of the Ising universality class. Further important progress was made on the determination of correlation functions [4. It was found, in particular, that the spin-spin correlator can be expressed, in the scaling limit, through the solution of a differential equation of Painlevé type [5]. Up to some generalisations, this remains the only non-trivial correlation function of quantum field theory to be exactly known.

These results refer to zero magnetic field, the Ising model in a field having never been solved

on the lattice. For this reason, A. Zamolodchikov's solution of the model with magnetic field, at the critical temperature, directly in the scaling limit, came as a major surprise for many [6]. Even more so since this solution consisted of a long list of scattering amplitudes for eight different species of relativistic particles. The most striking aspect of Zamolodchikov's work, however, was that, apart from its consequences for the Ising model, it actually implied new exact results for all the universality classes of critical behaviour in two dimensions. In fact, if conformal field theory had given a complete description of critical points [7, now it was shown that some renormalisation group trajectories flowing out of each critical point correspond to exactly solvable (integrable) quantum field theories. These describe particular directions in the scaling region of statistical models which, in general, are not solvable as long as the nonuniversal lattice details are not eliminated through the scaling limit. Specific realisations of a given universality class, however, can be solvable already on the lattice. In particular, a solvable lattice model yielding the same scaling limit of the Ising model in a magnetic field at critical temperature was found in $[$ ]

Dealing directly with the scaling limit, integrable quantum field theory is the most effective tool for extracting exact information about universality classes. In this context, 'integrable' 
means that the relativistic scattering theory associated to the quantum field theory can be determined exactly. Once this has been done, the next task is that of bridging the gap between the scattering solution and the quantities of more direct interest for statistical mechanics. In this article we review the results obtained through this approach for the universality class of the Ising model in a magnetic field, on the infinite plane. Although we refrain from making reference to other models, we stress the general reasons which allow to obtain similar results for the other universality classes in two dimensions. Since we always work in the continuum limit, we take care of comparing the field theoretical predictions for the universal quantities with the available lattice estimates.

The article is organised as follows. In the next section we recall the definition of the Ising model on the lattice before turning to the field theoretical description of the critical point and the scaling region around it. In section 3 we review the origin of integrable quantum field theories, their solution in the scattering framework and the form factor approach to the computation of correlation functions. All this is illustrated in practice through the application to the integrable directions of the Ising field theory in section 4, where the known results for the purely thermal case and the recent advances for the magnetic case are presented within the same framework. In particular, we discuss the determination of form factors in the magnetic case [9, 10]. Section 5 illustrates how the recent field theoretical results reflect onto the traditional way of characterising critical behaviour and allow to complete the list of canonical amplitude ratios for the Ising universality class. Finally, in section 6 , we briefly discuss how to exploit the integrable directions for a more general investigation of the scaling region and, in particular, review few basic results on the evolution of the particle spectrum in the Ising field theory.

\section{Ising field theory in two dimensions}

The Ising model is defined on the lattice by the reduced Hamiltonian

$$
E=-\frac{1}{T} \sum_{\langle i, j\rangle} \sigma_{i} \sigma_{j}-H \sum_{i} \sigma_{i},
$$

where $\sigma_{i}= \pm 1$ is the spin variable at the $i$-th site and the first sum is taken over nearest neighbours; the couplings $T$ and $H$ are referred to as temperature and magnetic field, respectively. The expectation value of any lattice variable $\mathcal{O}$ is given by

$$
\langle\mathcal{O}\rangle=\frac{1}{Z} \sum_{\left\{\sigma_{i}\right\}} \mathcal{O} e^{-E},
$$

where

$$
Z=\sum_{\left\{\sigma_{i}\right\}} e^{-E}
$$

is the partition function. We will always refer to the two-dimensional ferromagnetic $(T>0)$ case in the following. 
Consider the case $H=0$. The Hamiltonian is then invariant under the change of sign of all spins. This spin reversal symmetry is broken spontaneously when $T$ is smaller than a critical value $T_{c}$ for which a second order phase transition takes place [2]. The critical point divides the temperature axis into a high-temperature, disordered phase, and a low-temperature phase where a spontaneous magnetisation exists. The two phases are related by a duality transformation [1].

No global symmetry is left in the model when $H \neq 0$, and that located at $(T, H)=\left(T_{c}, 0\right)$ in the $T-H$ plane is the only critical point in the model. Since changing the sign of $H$ simply amounts to a global spin reversal transformation, it is sufficient to refer to the case $H \geq 0$.

The correlation length $\xi$ diverges at a second order phase transition point and remains much larger than the lattice spacing in a neighbourhood of this point in coupling space. In the scaling region in which $T \rightarrow T_{c}, H \rightarrow 0$, the system can effectively be considered as translationally and rotationally invariant, and quantum field theory provides a continous description suitable for the investigation of the universal properties (see e.g. [12]).

In particular, the behaviour of the critical point on scales much larger than the lattice spacing is described by a massless (mass $\sim 1 / \xi$ ) field theory invariant under scale transformations and, actually, under the larger group of conformal transformations. In two dimensions conformal symmetry is infinite dimensional and, for this reason, conformal field theories are exactly solved 7]. As it should be, the Ising critical point is described by the simplest (i.e. with the smallest operator content) conformal field theory satisfying the requirement of reflection positivity [13. This theory contains three fundamental (primary) operators which are invariant (scalar) under rotations, namely the identity $I$, the spin $\sigma(x)$ and the energy $\varepsilon(x)\left(x=\left(x_{1}, x_{2}\right)\right.$ denotes a point on the plane). The spin and energy operators are the continous version of the lattice variables $\sigma_{i}$ and $\sum_{j} \sigma_{i} \sigma_{j}$ ( $j$ nearest neighbour of $i$ ), respectively. Each primary operator possesses an infinite number of 'descendents', the simplest example being provided by the derivatives of the primaries. A primary and its descendents share the same internal symmetry properties and form a 'conformal family'.

In a conformal field theory the product of two operators $\Phi_{1}$ and $\Phi_{2}$ (to be thought inside a correlation function) can be expanded over a complete basis made of an infinite number of operators $A_{k}$ in the form

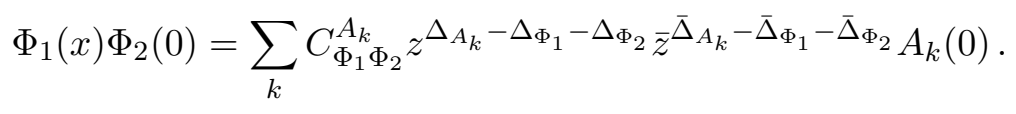

Here $z=x_{1}+i x_{2}$ and $\bar{z}=x_{1}-i x_{2}$ are complex coordinates, the $C_{\Phi_{1} \Phi_{2}}^{A_{k}}$ 's are called structure constants and $\Delta_{\Phi}$ and $\bar{\Delta}_{\Phi}$ are the conformal dimensions of an operator $\Phi(x)$. The scaling dimension

$$
X_{\Phi}=\Delta_{\Phi}+\bar{\Delta}_{\Phi}
$$

and the euclidean spin

$$
s_{\Phi}=\Delta_{\Phi}-\bar{\Delta}_{\Phi}
$$

determine the behaviour of the operator under scale transformations and rotations, respectively. 
The operators with a definite scaling dimension are called scaling operators. A scalar operator has $s_{\Phi}=0$.

While the scaling dimension of the identity operator vanishes, the two non-trivial primary operators in Ising field theory have dimensions $X_{\sigma}=1 / 8$ and $X_{\varepsilon}=1$. These two numbers determine all the critical exponents of the Ising universality class (see section 5 ). The conformal dimensions of the descendent operators differ by positive integers from those of the corresponding primary.

The structure of the operator product expansion in the Ising conformal theory can be symbolically expressed as

$$
\begin{aligned}
& \sigma \times \sigma \sim[I]+[\varepsilon] \\
& \sigma \times \varepsilon \sim[\sigma] \\
& \varepsilon \times \varepsilon \sim[I],
\end{aligned}
$$

where the square brackets indicate the appearence of a whole conformal family on the r.h.s. Clearly, this structure is compatible with the fact that the conformal families $[I]$ and $[\varepsilon]$ are even under spin reversal while $[\sigma]$ is odd.

The Ising critical point is left invariant by the duality transformation which exchanges the high- and low-temperature phases at $H=0$. While the energy operator $\varepsilon(x)$, which drives the model away from criticality along the thermal axis, changes sign under duality (this is why $[\varepsilon]$ does not appear in the last of (2.7) $)$, such a transformation maps $\sigma(x)$ onto a disorder operator $\mu(x)$ [14. The operators $\sigma$ and $\mu$ have the same scaling dimension $1 / 8$, but are mutually nonlocal, in the following sense. Two operators $\Phi_{1}$ and $\Phi_{2}$ are said to be mutually local if their product is unchanged when one of them is taken once around the other on the plane (i.e. under the analytic continuation $z \rightarrow e^{2 i \pi} z, \bar{z} \rightarrow e^{-2 i \pi} \bar{z}$ in (2.4) $)$. Of course this amounts to a statement about the single-valuedness of correlation functions involving the two operators. An operator is said to be local if it is local with respect to itself. It can be shown that the local operators (the only ones of interest for us) are those with integer or half-integer euclidean spin. The mildest type of mutual non-locality (called semi-locality) corresponds to the case

$$
\left\langle\cdots \Phi_{1}\left(e^{2 i \pi} z, e^{-2 i \pi} \bar{z}\right) \Phi_{2}(0) \cdots\right\rangle=l_{\Phi_{1}, \Phi_{2}}\left\langle\cdots \Phi_{1}(z, \bar{z}) \Phi_{2}(0) \cdots\right\rangle
$$

where $l_{\Phi_{1}, \Phi_{2}}$ is a phase called semi-locality factor.

The leading term in the $x \rightarrow 0$ expansion of the product $\sigma \times \mu$ is 14 .

$$
\sigma(x) \mu(0) \sim|x|^{-1 / 4}[\sqrt{z} \psi(0)+\sqrt{\bar{z}} \bar{\psi}(0)]+\ldots,
$$

where $\psi$ and $\bar{\psi}$ have conformal dimensions $(\Delta, \bar{\Delta})=(1 / 2,0)$ and $(0,1 / 2)$, respectively (the terms omitted in the r.h.s. contain descendents of $\psi$ and $\bar{\psi})$. This means that taking $\sigma$ around $\mu$ once produces a minus sign, so that the two operators are semi-local with $l_{\sigma, \mu}=-1$. Similarly, $\psi$ and $\bar{\psi}$ are semi-local with respect to $\sigma$ and $\mu$ with the same semi-locality factor -1 .

Summarising, the three conformal families $[I],[\sigma]$ and $[\varepsilon]$ we originally considered form a 'local section' of the Ising conformal field theory, namely a maximal set of fields all mutually 


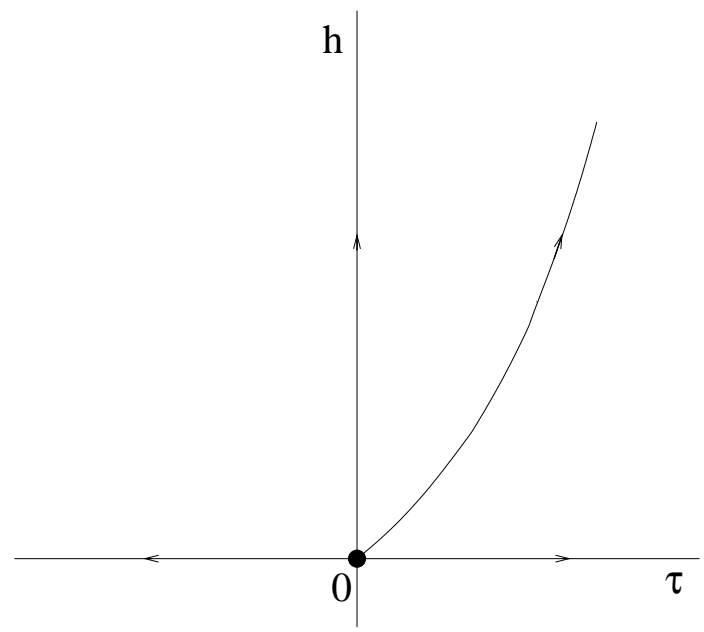

Figure 1: Coupling space of the Ising field theory (2.12). The oriented lines indicate some renormalisation group trajectories flowing out of the critical point at the origin. The integrable directions coincide with the principal axes. In our conventions $\tau>0$ corresponds to $T>T_{c}$.

local and closed under operator product expansion. Duality leads to consider a second local section differing from the first one by the substitution of $[\sigma]$ with $[\mu]$. A last local section is given by $[I],[\psi],[\bar{\psi}]$ and $[\varepsilon]$. All the conformal families together form the full space of local operators of the Ising conformal theory.

The operators $\psi$ and $\bar{\psi}$ are identified by their conformal dimensions as the two components of a neutral (Majorana) fermion. This is why the Ising conformal point is described by the free ${ }^{1}$ Hamiltonian (or euclidean action)

$$
\mathcal{A}_{0}=\frac{1}{2} \int d^{2} x(\psi \bar{\partial} \psi+\bar{\psi} \partial \bar{\psi})
$$

where $\partial=\partial_{z}=\left(\partial_{1}-i \partial_{2}\right) / 2$ and $\bar{\partial}=\partial_{\bar{z}}=\left(\partial_{1}+i \partial_{2}\right) / 2$. The conformal dimensions $(1 / 2,1 / 2)$ of the energy operator show that it is bilinear in the fermion components:

$$
\varepsilon \sim \bar{\psi} \psi
$$

$\sigma$ and $\mu$, on the contrary, are semi-local with respect to $\psi$ and $\bar{\psi}$ and give rise to the non-trivial sector of the theory (2.10).

The field theory describing the scaling region around the critical point is obtained by adding to the conformal action (2.10) the contributions of the operators conjugated to the termperature and the magnetic field, namely the energy and the spin operator, respectively. This leads to the Ising field theory

$$
\mathcal{A}=\mathcal{A}_{0}-\tau \int d^{2} x \varepsilon(x)-h \int d^{2} x \sigma(x)
$$

where

$$
\tau \sim M^{2-X_{\varepsilon}}=M
$$

\footnotetext{
${ }^{1}$ No interaction term preserving scale invariance can be formed.
} 


$$
h \sim M^{2-X_{\sigma}}=M^{15 / 8}
$$

are dimensional couplings measuring the deviation from critical temperature and the magnetic field, respectively. Here, $M \sim 1 / \xi$ denotes the mass scale associated to the breaking of scale invariance away from criticality. It is worth stressing that the action (2.12) is uniquely selected as the scaling limit of (2.1) by the fact that $\sigma$ and $\varepsilon$ are the only (non-constant) scalar relevant $^{2}$ operators in the local section of the operator space containing $\sigma$. Additional terms in (2.12) play a role only when trying to account for subleading terms in the expansion of lattice observables around the critical point (corrections to scaling). The field theory (2.12) describes a one-parameter family of renormalisation group trajectories flowing out of the critical point at $\tau=h=0$ (Figure 1) and labelled by the dimensionless quantity

$$
\eta=\frac{\tau}{|h|^{8 / 15}}
$$

The Ising model with $H=0$ is solvable on the lattice and then must be solvable in the scaling limit. In fact, (2.10) and (2.11) imply that for $h=0$ the action (2.12) describes a free massive fermion, the mass being proportional to $|\tau|$. For $H \neq 0$ the Ising model has never been solved on the lattice. A. Zamolodchikov showed that it is solvable directly in the scaling limit if $T=T_{c}$ [6]. This is a consequence of the fact that (2.12) with $\tau=0$ is an integrable quantum field theory. We summarise in the next section some generalities about integrable field theories before turning to the study of the integrable directions in the scaling Ising model (of course the free theory resulting from (2.12) when $h=0$ is a particularly simple case of integrability).

\section{Integrable quantum field theories}

\subsection{Conserved currents}

The notion of integrability is generally associated to the presence of an infinite number of conserved quantities. In two-dimensional quantum field theory a conservation law takes the form

$$
\bar{\partial} T_{s+1}=\partial \Theta_{s-1}
$$

where $T_{s+1}$ and $\Theta_{s-1}$ are local operators (currents) with spin $s+1$ and $s-1$, respectively. Any quantum field theory possesses the conservation law (3.1) with $s=1, T_{2}$ and $\Theta_{0}$ being components of the energy-momentum tensor. Are there theories allowing for additional, nontrivial conservation laws? The answer is obviously affirmative for the conformal theories: any descendent $T_{s}$ of the identity with conformal dimensions $(s, 0)$ is a local operator satisfying (3.1) with zero on the r.h.s. Of course, this is a direct consequence of the infinite dimensional character of conformal symmetry in two dimensions. It is then natural to ask whether any conservation law (other than that of energy and momentum) can survive when a non-scale-invariant theory is

\footnotetext{
${ }^{2}$ In the renormalisation group language, an operator is relevant if its scaling dimension is smaller than the space dimensionality ( 2 in our case).
} 
obtained as a perturbation of a conformal action $\mathcal{A}_{C F T}$ by a relevant operator $\Phi$, namely when considering the action

$$
\mathcal{A}=\mathcal{A}_{C F T}-g \int d^{2} x \Phi(x)
$$

In this case the original (conformal) conservation laws get modified into [6]

$$
\bar{\partial} T_{s}=g R_{s-1}^{(1)}+\cdots+g^{n} R_{s-1}^{(n)}+\cdots,
$$

where $R_{s-1}^{(n)}$ are operators with conformal dimensions $(s-n(1-\Delta), 1-n(1-\Delta))$ (the perturbing operator $\Phi$ has dimensions $(\Delta, \Delta), \Delta<1)$. It follows that in any theory with a spectrum of conformal dimensions bounded from below the r.h.s. of (3.3) can only contain a finite number of terms. Moreover, the operators $R_{s-1}^{(n)}$ with $n>1$ can be accomodated within the operator space of a theory with a discrete spectrum of conformal dimensions only if special relations between the dimensions are fulfilled. Hence, in a generic case, the r.h.s. of (3.3) contains only the operator $R_{s-1}^{(1)}$, which is a descendent of the perturbing operator $\Phi$. Thus the issue of conservation away from criticality is reduced to establishing under which conditions (if any) the operator $g R_{s-1}^{(1)}$ can be written in a total derivative form $\partial \Theta_{s-2}$. The complete characterisation of the operator space provided by conformal field theory ${ }^{3}$ allows the identification of sufficient conditions through a so-called 'counting argument' [6]. This exploits notions of conformal field theory which are not required in the remainder of this article, and we prefer to directly state the result of the analysis, which is remarkable: several non-trivial conservation laws (expected to be the first representatives of infinite series) can be found for a number of different perturbations of essentially all the known conformal points in two-dimensions; each integrable direction in coupling space is characterised by a specific set of values of the spin $s$ for which a conservation law of the form (3.1) is present.

For the Ising field theory (2.12), in particular, the counting argument implies integrability when $\tau=0$ and, of course, when $h=0$. The theory is not integrable when both couplings are different from zero [15, 16].

\section{$3.2 \quad$ Scattering theory}

The field theory obtained by perturbing a conformal theory by one or more relevant operators normally develops a finite correlation length and admits a description in terms of massive particles. These particles propagate in a space with one spatial and one time dimension, related by analytic continuation to imaginary time to the euclidean plane we have in mind for the applications to equilibrium statistical mechanics.

Integrability induces major simplifications in the scattering of relativistic particles (see [17] and references therein). Call

$$
P_{s}=\int_{-\infty}^{+\infty} d x_{1}\left[T_{s+1}(x)+\Theta_{s-1}(x)\right]
$$

\footnotetext{
${ }^{3}$ It is generally assumed that the operator spaces at the conformal point and in the perturbed theory (3.2) are isomorphic.
} 


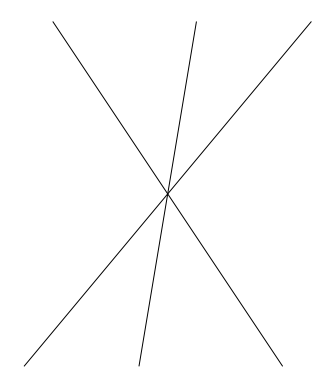

(a)

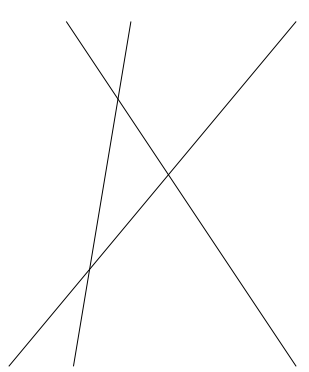

(b)

Figure 2: Space-time diagrams for a three-particle scattering process. A genuine three-body collision (a) or a sequence of widely separated two-body collisions (b) can be obtained by a suitable choice of initial conditions on the wave packets.

the spin $s>0$ conserved quantities, $P_{1}$ being the sum of energy and momentum. Also, denote by $A_{a}\left(p^{\mu}\right)$ a particle of type $a$ and energy-momentum $p^{\mu}=\left(p^{0}, p^{1}\right)$ satisfying the mass shell condition

$$
p^{\mu} p_{\mu}=\left(p^{0}\right)^{2}-\left(p^{1}\right)^{2}=m_{a}^{2}
$$

A conserved quantity acts as

$$
P_{s}\left|A_{a_{1}}\left(p_{1}^{\mu}\right) \ldots A_{a_{n}}\left(p_{n}^{\mu}\right)\right\rangle_{\text {in (out) }}=\left(\sum_{k=1}^{n} \omega_{s}^{a_{k}}\left(p_{k}^{\mu}\right)\right)\left|A_{a_{1}}\left(p_{1}^{\mu}\right) \ldots A_{a_{n}}\left(p_{n}^{\mu}\right)\right\rangle_{\text {in (out) }}
$$

on the initial (final) state of a scattering process containing $n$ widely separated particles. The behaviour under euclidean rotations fixes the form of the one-particle eigenvalue to be

$$
\omega_{s}^{a}\left(p^{\mu}\right)=\kappa_{s}^{a} p^{s}
$$

with $p=p^{0}+p^{1}$ and $\kappa_{s}^{a}$ a constant. Then conservation means that

$$
\sum_{k=1}^{n} \omega_{s}^{a_{k}}\left(p_{k}^{\mu}\right)=\sum_{j=1}^{m} \omega_{s}^{b_{j}}\left(q_{j}^{\mu}\right)
$$

in a scattering process with $n$ particles $A_{a_{k}}\left(p_{k}^{\mu}\right)$ in the initial state and $m$ particles $A_{b_{j}}\left(q_{j}^{\mu}\right)$ in the final state. Since this is a system of an infinite number of equations (one for each conserved quantity $P_{s}$ ) for a finite number of unknowns (the energies and momenta of the outgoing particles), one concludes that in a scattering process of an integrable quantum field theory

i) the final set of energies and momenta coincides with the initial one.

This is a remarkable simplification of the relativistic scattering problem. There is, however, an additional important result whose origin can be understood through the following euristic $\operatorname{argument}^{4}\left[18\right.$. The conserved quantities $P_{s}$ can be seen as generators of space-time displacements on wave packets. While $P_{1}$ simply translates the trajectories of all particles by the same

\footnotetext{
${ }^{4}$ See [19] for a discussion in the axiomatic framework.
} 


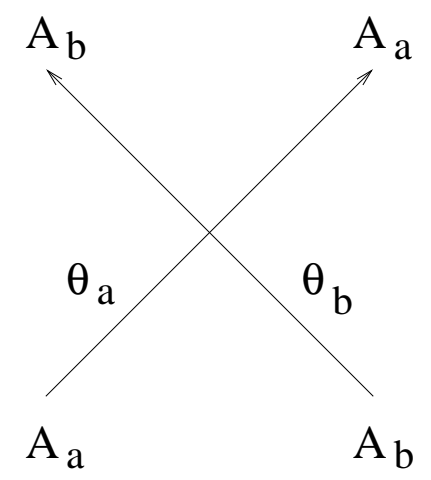

Figure 3: Space-time diagram associated to the scattering amplitude $S_{a b}\left(\theta_{1}-\theta_{2}\right)$; time runs upwards.

amount in space-time, for $s>1$ the amount of the shift depends on the momentum of each particle. Consider now a state containing three particles (wave packets) with different momenta and initial conditions chosen in such a way that they give rise to a genuine three-body interaction (Figure 2a). We can perform on this state a suitably chosen transformation generated by the $P_{s}$ with $s>1$ which shifts each trajectory by a different amount and resolves the three-body interaction into a sequence of three two-body collisions widely separated in space-time (Figure 2b). Since the $P_{s}$ are conserved, the transformation commutes with the time evolution and then the scattering amplitudes for the two processes coincide. The general conclusion is that in integrable quantum field theory

ii) any $n$-particle scattering amplitude factorises into the product of $n(n-1) / 2$ two-particle amplitudes.

We discussed the elasticity (i.e. absence of particle production) and factorisation of the scattering assuming the presence of an infinite number of conserved quantities. It has been shown, however, that the existence of one such a quantity besides energy-momentum is sufficient to arrive at the same conclusions [20].

As a consequence of factorisation, the determination of the $S$-matrix (i.e. the collection of all scattering amplitudes) in integrable quantum field theories reduces to that of the two-particle amplitudes. For the sake of simplicity we restrict our discussion to the case of neutral particles, which is enough for dealing with the Ising model. Then the two-particle amplitudes can be defined through the relation

$$
\left|A_{a}\left(\theta_{1}\right) A_{b}\left(\theta_{2}\right)\right\rangle=S_{a b}\left(\theta_{1}-\theta_{2}\right)\left|A_{b}\left(\theta_{2}\right) A_{a}\left(\theta_{1}\right)\right\rangle,
$$

where we introduced the rapidity variables parameterising on-shell momenta as

$$
\left(p^{0}, p^{1}\right)=\left(m_{a} \cosh \theta, m_{a} \sinh \theta\right)
$$

for a particle of mass $m_{a}$. Particles are ordered with rapidities decreasing (increasing) from left to right in initial (final) states. The dependence of the amplitudes on rapidity differences is a consequence of Lorentz invariance. A pictorial representation of a two-particle scattering 


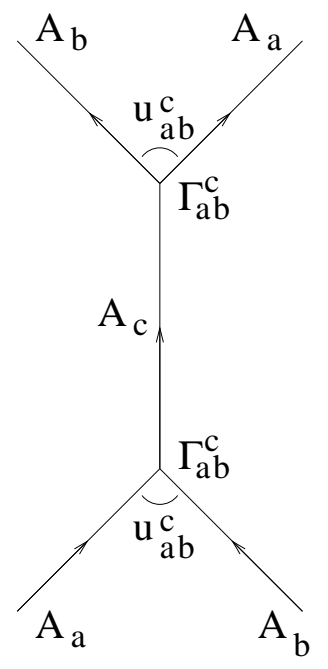

Figure 4: Simple pole diagram associated to equation (3.13).

amplitude is shown in Figure 3. Double application of (3.9) together with $S_{a b}(\theta)=S_{b a}(\theta)$ yield the unitarity relation

$$
S_{a b}(\theta) S_{a b}(-\theta)=1
$$

The crossing symmetry relation

$$
S_{a b}(\theta)=S_{a b}(i \pi-\theta)
$$

is a general property of relativistic scattering [21].

The scattering amplitudes $S_{a b}(\theta)$ are meromorphic functions of the rapidity difference. A simple pole with residue

$$
S_{a b}\left(\theta \simeq i u_{a b}^{c}\right) \simeq \frac{i\left(\Gamma_{a b}^{c}\right)^{2}}{\theta-i u_{a b}^{c}}
$$

and $u_{a b}^{c} \in(0, \pi)$ corresponds to a bound state with mass square

$$
m_{c}^{2}=m_{a}^{2}+m_{b}^{2}+2 m_{a} m_{b} \cos u_{a b}^{c}
$$

propagating in the $A_{a} A_{b}$ scattering channel. A three-particle coupling $\Gamma_{a b}^{c}$ is associated to each vertex of the corresponding diagram in Figure 4. It follows from (3.14) that

$$
\bar{u}_{a b}^{c}=\pi-u_{a b}^{c}
$$

is the angle opposite to $m_{c}$ in a triangle with sides of length $m_{a}, m_{b}, m_{c}$. This implies the relation

$$
u_{a b}^{c}+u_{b c}^{a}+u_{c a}^{b}=2 \pi .
$$

As a consequence of (3.12), a crossed channel pole with negative residue at $\theta=i\left(\pi-u_{a b}^{c}\right)$ goes along with each pole (3.13).

The general meromorphic solutions of (3.11) and (3.12) are of the form 22

$$
S_{a b}(\theta)= \pm \prod_{\alpha \in \mathcal{A}_{a b}} t_{\alpha}(\theta)
$$




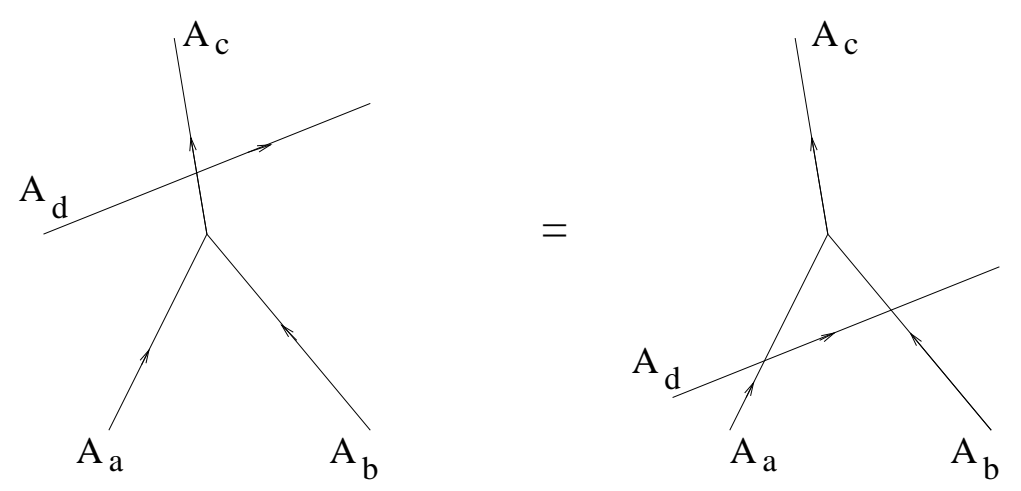

Figure 5: Pictorial representation of the bootstrap equation (3.21).

with

$$
t_{\alpha}(\theta)=\frac{\tanh \frac{1}{2}(\theta+i \pi \alpha)}{\tanh \frac{1}{2}(\theta-i \pi \alpha)} .
$$

Real values of $\alpha$ between 0 and 1 correspond to the bound state poles (3.13). Important constraints on the bound state structure come from the fact that an infinite number of quantities besides energy and momentum has to be conserved at each three-particle vertex [6]. In the vicinity of a bound state pole we can write

$$
\left|A_{a}\left(\theta+i \bar{u}_{c a}^{b}-\epsilon\right) A_{b}\left(\theta-i \bar{u}_{b c}^{a}+\epsilon\right)\right\rangle \sim \frac{1}{\epsilon}\left|A_{c}(\theta)\right\rangle .
$$

Applying $P_{s}$ to both sides and equating the eigenvalues gives

$$
\kappa_{s}^{a} m_{a}^{s} e^{i s \bar{u}_{c a}^{b}}+\kappa_{s}^{b} m_{b}^{s} e^{-i s \bar{u}_{b c}^{a}}=\kappa_{s}^{c} m_{c}^{s} .
$$

Integrability in presence of bound states also provides functional relations between different amplitudes. Indeed, we can consider the scattering of a particle $A_{d}$ with a resonant pair $A_{a} A_{b}$ and exploit the freedom of translating the particle trajectories to obtain (Figure 5)

$$
S_{d c}(\theta)=S_{d a}\left(\theta-i \bar{u}_{a c}^{b}\right) S_{d b}\left(\theta+i \bar{u}_{b c}^{a}\right) .
$$

These equations are known as 'bootstrap' equations for the following reason. Suppose that the use of (3.20) plus other considerations ends up in an educated guess for the scattering amplitudes of the 'elementary' (lightest) particles of the theory. Then one can use (3.21) with $A_{a}, A_{b}, A_{d}$ 'elementary' particles and $A_{c}$ one of their bound states to determine the amplitude $S_{d c}$. Each amplitude computed in this way may present poles corresponding to new particles, in which case the use of (3.21) is iterated. Once this bootstrap procedure consistently reaches a point where no new particles are generated, one is left with the exact solution of an integrable quantum field theory (see [15] for a series of examples).

\subsection{Correlation functions}

Having the $S$-matrix is not enough for statistical mechanical applications; correlation functions are needed. These can be expressed in the form of spectral sums over complete sets of particle 


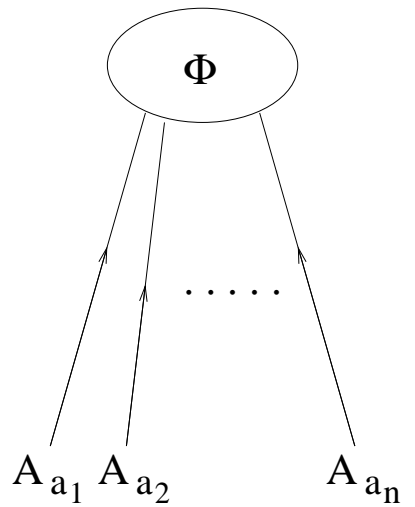

Figure 6: Pictorial representation of an $n$-particle form factor.

states. For example, the (euclidean) correlator of two scalar operators reads ${ }^{5}$

$$
\left\langle\Phi_{1}(x) \Phi_{2}(0)\right\rangle=\sum_{n=0}^{\infty} \frac{1}{(2 \pi)^{n}} \int_{\theta_{1}>\cdots>\theta_{n}} d \theta_{1} \cdots d \theta_{n} F_{a_{1} \ldots a_{n}}^{\Phi_{1}}\left(\theta_{1}, \ldots, \theta_{n}\right)\left[F_{a_{1} \ldots a_{n}}^{\Phi_{2}}\left(\theta_{1}, \ldots, \theta_{n}\right)\right]^{*} e^{-|x| E_{n}},
$$

where

$$
E_{n}=\sum_{k=1}^{n} m_{a_{k}} \cosh \theta_{k}
$$

and the matrix elements (Figure 6)

$$
F_{a_{1} \ldots a_{n}}^{\Phi}\left(\theta_{1}, \ldots, \theta_{n}\right)=\left\langle 0|\Phi(0)| A_{a_{1}}\left(\theta_{1}\right) \ldots A_{a_{n}}\left(\theta_{n}\right)\right\rangle
$$

are called form factors $(|0\rangle$ is the vacuum state).

The form factors can be computed exactly in integrable quantum field theory. They are subject to a number of equations [23, 24] which for the case of neutral particles we are discussing read

$$
\begin{aligned}
& F_{a_{1} \ldots a_{n}}^{\Phi}\left(\theta_{1}+\Lambda, \ldots, \theta_{n}+\Lambda\right)=e^{s \Lambda} F_{a_{1} \ldots a_{n}}^{\Phi}\left(\theta_{1}, \ldots, \theta_{n}\right) \\
& F_{a_{1} \ldots a_{i} a_{i+1} \ldots a_{n}}^{\Phi}\left(\theta_{1}, \ldots, \theta_{i}, \theta_{i+1}, \ldots, \theta_{n}\right)= \\
& \quad S_{a_{i} a_{i+1}}\left(\theta_{i}-\theta_{i+1}\right) F_{a_{1} \ldots a_{i+1} a_{i} \ldots a_{n}}^{\Phi}\left(\theta_{1}, \ldots, \theta_{i+1}, \theta_{i}, \ldots, \theta_{n}\right) \\
& \operatorname{Res}_{\theta_{a}-\theta_{b}=i u_{a b}^{c}} F_{a b a_{1} \ldots a_{n}}^{\Phi}\left(\theta_{a}, \theta_{b}, \theta_{1}, \ldots, \theta_{n}\right)=i \Gamma_{a b}^{c} F_{c a_{1} \ldots a_{n}}^{\Phi}\left(\theta_{c}, \theta_{1}, \ldots, \theta_{n}\right) \\
& F_{a_{1} \ldots a_{n}}^{\Phi}\left(\theta_{1}+2 i \pi, \theta_{2}, \ldots, \theta_{n}\right)=l_{\Phi, \phi_{a_{1}}} F_{a_{2} \ldots a_{n} a_{1}}^{\Phi}\left(\theta_{2}, \ldots, \theta_{n}, \theta_{1}\right) \\
& \operatorname{Res}_{\theta^{\prime}=\theta+i \pi} F_{a b a_{1} \ldots a_{n}}^{\Phi}\left(\theta^{\prime}, \theta, \theta_{1}, \ldots, \theta_{n}\right)= \\
& \qquad i \delta_{a b}\left(1-l_{\Phi, \phi_{a}} \prod_{j=1}^{n} S_{a_{j} a}\left(\theta_{j}-\theta\right)\right) F_{a_{1} \ldots a_{n}}^{\Phi}\left(\theta_{1}, \ldots, \theta_{n}\right)
\end{aligned}
$$

The first equation expresses Lorentz covariance for a spin $s$ operator, while the second is a consequence of (3.9) and factorisation of multi-particle scattering. The third equation

\footnotetext{
${ }^{5}$ Particle states are normalised through the condition $\left\langle A_{a}\left(\theta_{1}\right) \mid A_{b}\left(\theta_{2}\right)\right\rangle=2 \pi \delta_{a b} \delta\left(\theta_{1}-\theta_{2}\right)$.
} 
says that the form factors inherit the direct channel bound state poles of the $S$-matrix $\left(\theta_{c}=\right.$ $\left.\left(\bar{u}_{b c}^{a} \theta_{a}+\bar{u}_{c a}^{b} \theta_{b}\right) / u_{a b}^{c}\right)$.

Within the framework of relativistic scattering theory, a crossing process corresponds to trading a particle of energy-momentum $p^{\mu}$ in the initial (final) state with an antiparticle of energy-momentum $-p^{\mu}$ in the final (initial) state [21. The inversion of energy-momentum corresponds to an $i \pi$ shift in the rapitidy parameterisation. Hence, equation (3.28) shows that the (kinematically immaterial) double crossing of a particle corresponds to a reordering of rapidities. If the operator $\phi_{a}$ which creates the particle ${ }^{6} A_{a}$ is non-local (semi-local, for simplicity) with respect to $\Phi$, the double crossing of this particle also produces a phase factor $l_{\Phi, \phi_{a}} \neq 1$ (recall the definition (2.8) 25].

Finally, equation (3.29) expresses the fact that two identical particles with opposite energymomentum can annihilate. This gives rise to a pole whose residue reflets the fact that the annihilation of adjacent particles can take place either directly or through the analytic continuation (3.28).

The form factor equations listed above are satisfied by all the operators of the theory and then admit an infinite number of solutions. Identifying the solution corresponding to a given operator is a central problem in this approach. The following argument turns out to be quite helpful in this respect. Consider a scalar operator $\Phi$ with scaling dimension $X_{\Phi}$ in a massive theory. Since

$$
\langle\Phi(x) \Phi(0)\rangle \sim \frac{1}{|x|^{2 X_{\Phi}}}, \quad|x| \rightarrow 0
$$

we know that ${ }^{7}$

$$
M_{p}=\int d^{2} x|x|^{p}\langle\Phi(x) \Phi(0)\rangle_{c}
$$

is finite only if

$$
p+2>2 X_{\Phi} .
$$

Once we use (3.22) to expand the correlator in (3.31) and perform the spatial intergration, we are left with a series of $n$-fold integrals over rapidities with positive integrands (moduli squared of form factors). Provided (3.32) holds, each term of the series has to be finite, what implies an upper bound on the asymptotic behaviour of the form factors. One can check that chosing the value of $p$ yielding the most stringent bound gives the final result 9 ]

$$
\lim _{\left|\theta_{i}\right| \rightarrow \infty} F_{a_{1} \ldots a_{n}}^{\Phi}\left(\theta_{1}, \ldots, \theta_{n}\right) \sim \exp \left(Y_{\Phi}\left|\theta_{i}\right|\right)
$$

with

$$
Y_{\Phi} \leq \frac{X_{\Phi}}{2}
$$

Explicit expressions for all the form factors of a given operator can be obtained in many cases. The problem of summing series like (3.22), however, remains unsolved for interacting theories.

\footnotetext{
${ }^{6}$ Any operator with $\left\langle 0\left|\phi_{a}\right| A_{a}\right\rangle \neq 0$ can be taken as creating operator. In order to get rid of spin factors, we always choose $\phi_{a}$ to be scalar.

${ }^{7}$ We denote by $\langle\cdots\rangle_{c}$ the connected correlators.
} 
Due to the exponential dumping factor, (3.22) is a large distance expansion. A complementary short distance expansion is provided by perturbation theory around the conformal point [26]. Direct comparison shows that both expansions converge very rapidly and that the first few terms provide a very good matching at the intermediate scales where the crossover from the short distance power law behaviour to the large distance exponetial decay takes place.

Integrated correlators like (3.31) are what one needs to compute in many physical applications (see e.g. section 5). In these cases the contribution of short distances is suppressed by the powers of $|x|$ in the integrand, and the form factor expansion alone is sufficient to obtain precise estimates. A check of the convergence can be done through sum rules allowing to recover conformal data from the off-critical correlation functions. Conformal theories in two dimensions are labelled by a number $C$ called 'central charge' [7. It is a consequence of Zamolodchikov's $C$ theorem [27] that the central charge of the conformal limit of a massive theory can be expressed as 28 ]

$$
C=\frac{3}{4 \pi} \int d^{2} x|x|^{2}\langle\Theta(x) \Theta(0)\rangle_{c},
$$

where $\Theta(x)$ is the trace of the energy-momentum tensor. Similarly, the scaling dimension of a relevant operator $\Phi(x)$ is computable through the sum rule [29].

$$
X_{\Phi}=-\frac{1}{2 \pi\langle\Phi\rangle} \int d^{2} x\langle\Theta(x) \Phi(0)\rangle_{c}
$$

\section{Integrable directions in the scaling Ising model}

\subsection{Zero magnetic field}

As we have already seen, the scaling limit of the Ising model without magnetic field is described by the action (2.12) with $h=0$, namely by a theory of free neutral fermions with mass $m$ proportional to $|\tau|$. Depending on the sign of $\tau$, this theory describes the ordered or disordered phase. To be definite, we will work in the high-temperature phase $(\tau>0)$ and will use the duality transformations $\varepsilon \leftrightarrow-\varepsilon$ and $\sigma \leftrightarrow \mu$ to obtain the results in the ordered phase.

The free fermionic theory provides a particularly simple example of integrable field theory. It contains a single neutral particle $A$ with fermionic statistics and $S$-matrix equal to 1 . In order to fit the conventions on form factors of the previous section, however, we will think of the particle as created by a bosonic operator and absorb the anticommutativity into the $S$-matrix, so that the two-particle scattering amplitude reads

$$
S=-1
$$

In the high-temperature phase we are considering, the particles correspond to local spin excitations and the spin operator $\sigma$ is naturally identified as the scalar creation operator ${ }^{8}$.

\footnotetext{
${ }^{8}$ In the low-temperature phase the excitations are kinks interpolating between the two degenerate vacua. Such topologic excitations are non-local in the spin degrees of freedom and the creation operator corresponding to the amplitude 4.1] is the disorder operator $\mu$.
} 
For the present case in which we have only one type of particle the notation for form factors can be simplified to

$$
F_{n}^{\Phi}\left(\theta_{1}, \ldots, \theta_{n}\right)=\left\langle 0|\Phi(0)| A\left(\theta_{1}\right) \ldots A\left(\theta_{n}\right)\right\rangle
$$

so that the form factor equations become

$$
\begin{aligned}
& F_{n}^{\Phi}\left(\theta_{1}+\Lambda, \ldots, \theta_{n}+\Lambda\right)=e^{s \Lambda} F_{n}^{\Phi}\left(\theta_{1}, \ldots, \theta_{n}\right) \\
& F_{n}^{\Phi}\left(\theta_{1}, \ldots, \theta_{i}, \theta_{i+1}, \ldots, \theta_{n}\right)=-F_{n}^{\Phi}\left(\theta_{1}, \ldots, \theta_{i+1}, \theta_{i}, \ldots, \theta_{n}\right) \\
& F_{n}^{\Phi}\left(\theta_{1}+2 i \pi, \theta_{2}, \ldots, \theta_{n}\right)=l_{\Phi, \sigma} F_{n}^{\Phi}\left(\theta_{2}, \ldots, \theta_{n}, \theta_{1}\right) \\
& \operatorname{Res}_{\theta^{\prime}=\theta+i \pi} F_{n+2}^{\Phi}\left(\theta^{\prime}, \theta, \theta_{1}, \ldots, \theta_{n}\right)=i\left[1-(-1)^{n} l_{\Phi, \sigma}\right] F_{n}^{\Phi}\left(\theta_{1}, \ldots, \theta_{n}\right)
\end{aligned}
$$

(equation (3.27) plays no role in absence of bound states). We know from the discussion of section 2 that

$$
l_{\sigma, \sigma}=l_{\varepsilon, \sigma}=-l_{\mu, \sigma}=1 .
$$

The solutions 30]

$$
\begin{aligned}
& F_{n}^{\Theta}\left(\theta_{1}, \ldots, \theta_{n}\right)=c_{1} m F_{n}^{\varepsilon}\left(\theta_{1}, \ldots, \theta_{n}\right)=-2 i \pi m^{2} \delta_{n, 2} \sinh \frac{\theta_{1}-\theta_{2}}{2} \\
& F_{2 n+1}^{\sigma}\left(\theta_{1}, \ldots, \theta_{2 n+1}\right)=i^{n} F_{1}^{\sigma} \prod_{i<j} \tanh \frac{\theta_{i}-\theta_{j}}{2} \\
& F_{2 n}^{\mu}\left(\theta_{1}, \ldots, \theta_{2 n}\right)=i^{n} F_{0}^{\mu} \prod_{i<j} \tanh \frac{\theta_{i}-\theta_{j}}{2}
\end{aligned}
$$

are the only ones satisfying the asymptotic bound (3.34) for the case of relevant scalar operators. This is a simple example of how the form factor approach reveals the operator content behind a scattering theory. A counting of solutions including descendents confirms the correspondence with the operator space of the conformal point 31]. In writing (4.8) we used the fact that, being the operator which breaks conformal invariance, $\varepsilon$ is proportional to the trace of the energy-momentum tensor ( $c_{1}$ is a dimensionless constant) for which the normalisation condition

$$
F_{a a}^{\Theta}(\theta+i \pi, \theta)=2 \pi m_{a}^{2}
$$

holds. The vanishing of the $F_{n}^{\varepsilon}$ with $n \neq 2$ matches the fact that the energy operator is bilinear in the free fermions. The results for $\sigma$ and $\mu$ are non-trivial and reflect the non-locality of these operators with respect to the fermions. The form factors of $\sigma$ with an even number of particles have to vanish because the particles are odd under $\sigma \rightarrow-\sigma$; the invariance of $\mu$ under this symmetry induces the vanishing of the $F_{2 n+1}^{\mu}$.

It follows from (4.8) that the form factor expansion of a two-point function involving the energy operator contains only one term. In particular, the sum rules (3.35) and (3.36) immediately give the exact results $^{9}\left(F_{0}^{\mu}=\langle\mu\rangle\right)$

$$
C=\frac{3}{2} \int_{0}^{\infty} d \theta \frac{\sinh ^{2} \theta}{\cosh ^{4} \theta}=\frac{1}{2}
$$

\footnotetext{
${ }^{9}$ The integral in (3.36) diverges for $\Phi=\varepsilon$. See [29] on this point.
} 


$$
X_{\mu}=\frac{1}{2 \pi} \int_{0}^{\infty} d \theta \frac{\sinh ^{2} \theta}{\cosh ^{3} \theta}=\frac{1}{8}
$$

Form factor series with an infinite number of terms are instead obtained for the correlators $\langle\sigma \sigma\rangle$ and $\langle\mu \mu\rangle$. Due to the particularly simple form of the matrix elements (4.9) and (4.10) these series can be resummed [32, 33. We quote here the final result of this procedure which, of course, reproduces that originally obtained in [5] from the lattice solution. The correlators can be written in the form

$$
\begin{gathered}
\langle\sigma(x) \sigma(0)\rangle=\langle\mu\rangle^{2} e^{-\Sigma(t)} \sinh \frac{1}{2} \chi(t / 2) \\
\langle\mu(x) \mu(0)\rangle=\langle\mu\rangle^{2} e^{-\Sigma(t)} \cosh \frac{1}{2} \chi(t / 2),
\end{gathered}
$$

where $t=m|x|$ and

$$
\Sigma(t)=\frac{1}{4} \int_{t / 2}^{\infty} d \rho \rho\left[\left(\partial_{\rho} \chi\right)^{2}-4 \sinh ^{2} \chi\right] .
$$

The function $\chi$ is the solution of the differential equation ${ }^{10}$

$$
\partial_{\rho}^{2} \chi+\frac{1}{\rho} \partial_{\rho} \chi=2 \sinh 2 \chi
$$

satisfying the asymptotic conditions

$$
\begin{aligned}
& \chi(\rho) \simeq-\ln \rho+\text { constant }, \quad \rho \rightarrow 0 \\
& \chi(\rho) \simeq \frac{2}{\pi} K_{0}(2 \rho), \quad \rho \rightarrow \infty .
\end{aligned}
$$

Both (4.14) and (4.15) behave as

$$
\frac{C_{I}}{|x|^{2 X_{\sigma}}}=\frac{C_{I}}{|x|^{1 / 4}}
$$

as $|x| \rightarrow 0$. When $|x| \rightarrow \infty$ the correlators decay exponentially: $\langle\sigma \sigma\rangle$ vanish while $\langle\mu \mu\rangle$ approaches $\langle\mu\rangle^{2}$. If the operators are normalised in such a way that $C_{I}=1$, then

$$
\langle\mu\rangle= \pm m^{1 / 8} 2^{1 / 12} e^{-1 / 8} A^{3 / 2},
$$

$A$ being the Glaisher constant

$$
A=2^{7 / 36} \pi^{-1 / 6} \exp \left[\frac{1}{3}+\frac{2}{3} \int_{0}^{\frac{1}{2}} d x \ln \Gamma(1+x)\right]=1.282427129 . .
$$

These results for the correlation functions of the scaling Ising model without magnetic field were also derived in 34 using the theory of monodromy preserving deformations of ordinary differential equations. Very recently a simpler derivation based on the Ward identities associated to the conservation laws of the free fermionic theory has been given in [35. The convergence of the form factor series was analysed numerically in 25. A study of four-point functions in the form factor approach can be found in [36].

\footnotetext{
${ }^{10}$ Equation (4.17) becomes a Painlevé III equation for the function $\eta=e^{-\chi}$.
} 


\subsection{Non-zero magnetic field at $T=T_{c}$}

As anticipated in section 2, the Ising field theory (2.12) with $h \neq 0$ is integrable only when $\tau=0$. The counting argument applied to this purely magnetic perturbation of the Ising conformal point shows that conserved quantities of the form (3.4) exist for spin $s=1,7,11,13,17,19$ [ 6 . These are expected to be the first representatives of the infinite set

$$
s=1,7,11,13,17,19,23,29(\bmod 30) .
$$

It can be noted that this set of numbers coincides with the exponents of the Lie algebra $E_{8}$ repeated modulo the Coxeter number of the algebra. The spectrum of conserved spins (4.23) and its relation with the algebra $E_{8}$ were first predicted by V. Fateev [37, 38].

Since the Ising field theory with non-zero magnetic field does not possess any internal symmetry, its mass spectrum is guaranteed to be non-degenerate. Hence we know that the two-particle scattering amplidutes of the integrable theory must be of the form (3.17). A. Zamolodchikov looked for the minimal solution ${ }^{11}$ of the bootstrap equations (3.21) satisfying the conservation equations (3.20) with the set of spin values (4.23) 6]. He found that this requirement leads to a bootstrap system closing on 8 particles $A_{a}(a=1, \ldots, 8)$ with masses

$$
\begin{aligned}
& m_{2}=2 m_{1} \cos \frac{\pi}{5}=(1.6180339887 . .) m_{1} \\
& m_{3}=2 m_{1} \cos \frac{\pi}{30}=(1.9890437907 . .) m_{1} \\
& m_{4}=2 m_{2} \cos \frac{7 \pi}{30}=(2.4048671724 . .) m_{1} \\
& m_{5}=2 m_{2} \cos \frac{2 \pi}{15}=(2.9562952015 . .) m_{1} \\
& m_{6}=2 m_{2} \cos \frac{\pi}{30}=(3.2183404585 . .) m_{1} \\
& m_{7}=4 m_{2} \cos \frac{\pi}{5} \cos \frac{7 \pi}{30}=(3.8911568233 . .) m_{1} \\
& m_{8}=4 m_{2} \cos \frac{\pi}{5} \cos \frac{2 \pi}{15}=(4.7833861168 . .) m_{1}
\end{aligned}
$$

in units of the lightest mass $m_{1}$. The scattering amplitudes can be written as

$$
S_{a b}(\theta)=\prod_{\gamma \in \mathcal{G}_{a b}}\left(t_{\gamma / 30}(\theta)\right)^{p_{\gamma}}
$$

in terms of the building blocks (3.18); the complete list of indices $\gamma$ and exponents $p_{\gamma}$ is given in Table 1. For example, the $A_{1} A_{1}$ and $A_{1} A_{2}$ amplitudes read

$$
\begin{aligned}
& S_{11}(\theta)=t_{2 / 3}(\theta) t_{2 / 5}(\theta) t_{1 / 15}(\theta) \\
& S_{12}(\theta)=t_{4 / 5}(\theta) t_{3 / 5}(\theta) t_{7 / 15}(\theta) t_{4 / 15}(\theta) .
\end{aligned}
$$

Equation (3.14) shows that the particles $A_{1}, A_{2}, A_{3}\left(A_{1}, A_{2}, A_{3}, A_{4}\right)$ appear as bound states in the $A_{1} A_{1}\left(A_{1} A_{2}\right)$ scattering channel.

\footnotetext{
${ }^{11}$ In general, the solution of the $S$-matrix equations of section 3.2 is not unique. There exists, however, a minimal solution possessing the smallest number of zeros and poles in the physical strip $\operatorname{Im} \theta \in(0, \pi)$.
} 


\begin{tabular}{|c|c|c|}
\hline & $b$ & $S_{a b}$ \\
\hline 1 & 1 & $\begin{array}{ccc}\mathbf{1} & \mathbf{2} & \mathbf{3} \\
(20) & (12)(2)\end{array}$ \\
\hline & & $\begin{array}{cccc}1 & 2 & 3 & 4 \\
(14) & (14) & (8)\end{array}$ \\
\hline & 2 & $\begin{array}{cccc}(24) & (18) & (14)(8) \\
\mathbf{1} & \mathbf{2} & \mathbf{4} & \mathbf{5}\end{array}$ \\
\hline 1 & 3 & $(29)(21)(13)(3)(11)^{2}$ \\
\hline & 4 & 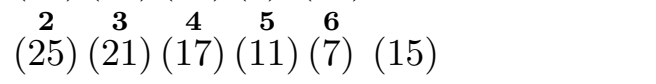 \\
\hline & 5 & 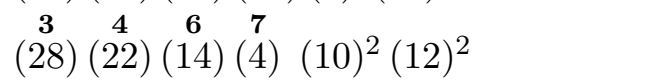 \\
\hline 1 & 6 & 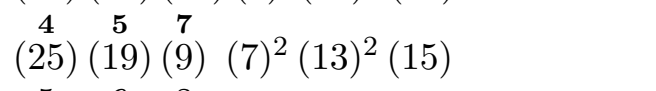 \\
\hline 1 & 7 & $\begin{array}{cccc}\mathbf{5} & \mathbf{6} & \mathbf{8} & \\
(27) & (23) & (5) & (9)^{2} \\
(11)^{2} & (13)^{2}(15)\end{array}$ \\
\hline & 8 & $(26)(16)^{3}(6)^{2}(8)^{2}(10)^{2}(12)^{2}$ \\
\hline 2 & 2 & 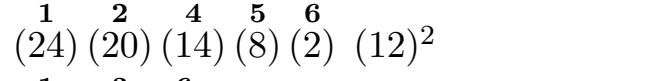 \\
\hline 2 & 3 & 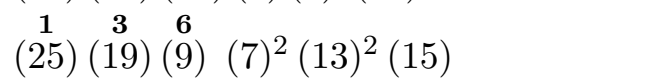 \\
\hline 2 & 4 & $(27)(23)(5)(9)^{2}(11)^{2}(13)^{2}(15)$ \\
\hline 2 & 5 & 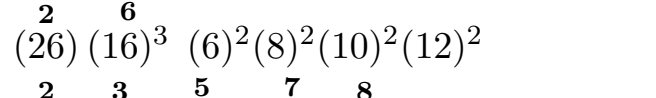 \\
\hline 2 & 6 & $(29)(25)(19)^{3}(13)^{3}(3)(7)^{2}(9)^{2}(15)$ \\
\hline 2 & 7 & 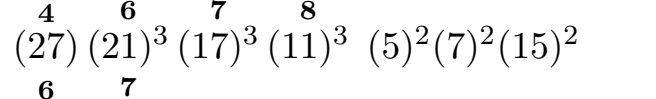 \\
\hline 2 & 8 & $(28)(22)^{3}(4)^{2}(6)^{2}(10)^{4}(12)^{4}(16)^{4}$ \\
\hline 3 & 3 & $(22)(20)^{3}(14)(12)^{3}(4)(2)^{2}$ \\
\hline & 4 & $\stackrel{\mathbf{1}}{\mathbf{5}} \underset{\mathbf{5}}{(\mathbf{6}})^{3}(6)^{2}(8)^{2}(10)^{2}(12)^{2}$ \\
\hline 3 & 5 & 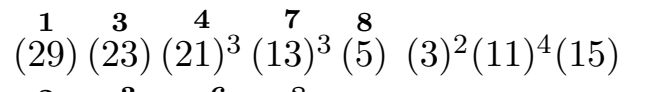 \\
\hline 3 & 6 & $\begin{array}{ccccc}\mathbf{2} & \mathbf{3} & \mathbf{6} & \mathbf{8} & \\
(26) & (24)^{3} & (18)^{3} & (8)^{3} & (10)^{2}(16)^{4}\end{array}$ \\
\hline 3 & 7 & 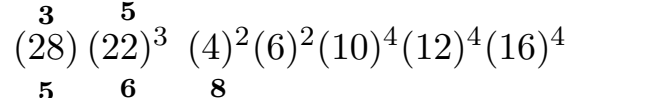 \\
\hline 3 & 8 & $(27)(25)^{3}(17)^{5}(7)^{4}(9)^{4}(11)^{2}(15)^{3}$ \\
\hline 4 & 4 & 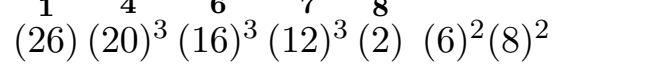 \\
\hline 4 & 5 & $\begin{array}{ccccc}\mathbf{1} & \mathbf{3} & \mathbf{5} & \mathbf{8} & \\
(27) & (23)^{3} & (19)^{3} & (9)^{3} & (5)^{2}(13)^{4}(15)^{2}\end{array}$ \\
\hline 4 & 6 & $\underset{(28)}{\mathbf{1}} \underset{(22}{\mathbf{4}})^{3}(4)^{2}(6)^{2}(10)^{4}(12)^{4}(16)^{4}$ \\
\hline 4 & 7 & $\begin{array}{ccccc}\mathbf{2} & \mathbf{4} & \mathbf{7} & \mathbf{8} & \\
(28) & (24)^{3} & (18)^{5} & (14)^{5} & (4)^{2}(8)^{4}(10)^{4}\end{array}$ \\
\hline 4 & 8 & $\begin{array}{cccc}\mathbf{4} & \mathbf{5} & \mathbf{7} & \\
(29) & (25)^{3} & (21)^{5} & (3)^{2}(7)^{4}(11)^{6}(13)^{6}(15)^{3}\end{array}$ \\
\hline
\end{tabular}




\begin{tabular}{|c|c|c|}
\hline & 5 & $\begin{array}{cccc}\mathbf{4} & \mathbf{5} & \mathbf{8} & \\
(22)^{3} & (20)^{5} & (12)^{5} & (2)^{2}(4)^{2}(6)^{2}(16)^{4}\end{array}$ \\
\hline & & $\begin{array}{cccc}1 & 2 & 7 & \end{array}$ \\
\hline 5 & 6 & $(27)(25)^{3}(17)^{5}(7)^{4}(9)^{4}(11)^{4}(15)^{3}$ \\
\hline 5 & 7 & 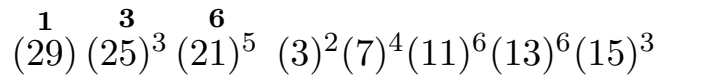 \\
\hline 5 & 8 & $\begin{array}{ccccc}\mathbf{3} & \mathbf{4} & \mathbf{5} & \mathbf{8} & \\
(28) & (26)^{3} & (24)^{5} & (18)^{7} & (8)^{6}(10)^{6}(16)^{8}\end{array}$ \\
\hline & 6 & $\begin{array}{cccc}\mathbf{3} & \mathbf{6} & \mathbf{8} & \\
(24)^{3} & (20)^{5} & (14)^{5} & (2)^{2}(4)^{2}(8)^{4}(12)^{6}\end{array}$ \\
\hline 6 & 7 & $\begin{array}{ccccc}\mathbf{1} & \mathbf{2} & \mathbf{5} & \mathbf{8} & \\
(28) & (26)^{3} & (22)^{5} & (16)^{7} & (6)^{4}(10)^{6}(12)^{6}\end{array}$ \\
\hline 6 & 8 & 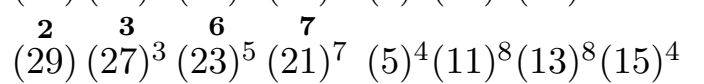 \\
\hline & 7 & $\begin{array}{ccc}\mathbf{2} & \mathbf{4} & \mathbf{7} \\
(26)^{3}(24)^{5}(20)^{7} & (2)^{2}(8)^{6}(12)^{8}(16)^{8}\end{array}$ \\
\hline 7 & 8 & 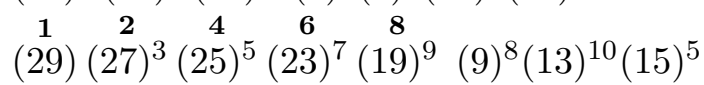 \\
\hline 8 & 8 & 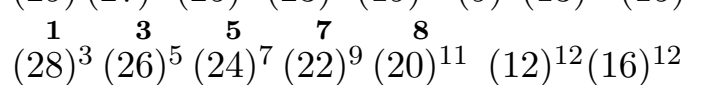 \\
\hline
\end{tabular}

Table 1: $S$-matrix of the scaling Ising model in a magnetic field at $T=T_{c}$. A factor $\left(t_{\gamma / 30}(\theta)\right)^{p_{\gamma}}$ in the amplitude $S_{a b}(\theta)$ corresponds to each term $(\gamma)^{p_{\gamma}}\left(p_{\gamma}=1\right.$ is omitted; the building blocks $t_{\alpha}(\theta)$ are defined in (3.18) $)$. The superscript $\mathbf{c}$ above $(\gamma)$ indicates that the pole at $\theta=i \pi \gamma / 30$ in the amplitude $S_{a b}(\theta)$ corresponds to the particle $A_{c}$ appearing as bound state in the $A_{a} A_{b}$ scattering channel.

Most of the amplitudes in Table 1 contain higher order poles that we did not discuss in the previous section. Within the framework of the analytic $S$-matrix, each singularity is expected to have a physical interpretation. The higher order poles of the scattering amplitudes were recognised in [39] (see also [40, 41]) as the singularities that in two dimensions are associated to processes in which more than one particle propagates in the intermediate state $^{12}$. Figures 7 and 8 show the processes of this kind leading to second and third order poles at $\theta=i \varphi$,

$$
\varphi=u_{a d}^{c}+u_{d b}^{e}-\pi
$$

in the amplitude $S_{a b}(\theta)$. The angle $\eta$ in Figure $7 \mathrm{a}$ is

$$
\eta=\pi-u_{c d}^{a}-u_{d e}^{b} \in[0, \pi)
$$

and $i \eta$ is the rapidity difference between the intermediate propagating particles $A_{c}$ and $A_{e}$; the diagram of Figure $7 \mathrm{~b}$ corresponds to the limiting case $\eta=0$. One has

$$
\begin{aligned}
S_{a b}(\theta \simeq i \varphi) & \simeq \frac{\left(\Gamma_{c d}^{a} \Gamma_{d e}^{b}\right)^{2} S_{c e}(i \eta)}{(\theta-i \varphi)^{2}} \\
S_{a b}(\theta \simeq i \varphi) & \simeq \frac{\Gamma_{c d}^{a} \Gamma_{d e}^{b} \Gamma_{c f}^{b} \Gamma_{f e}^{a}}{(\theta-i \varphi)^{2}}
\end{aligned}
$$

\footnotetext{
${ }^{12}$ In four dimensions these processes give rise to anomalous thresholds rather than poles [21].
} 
(a)

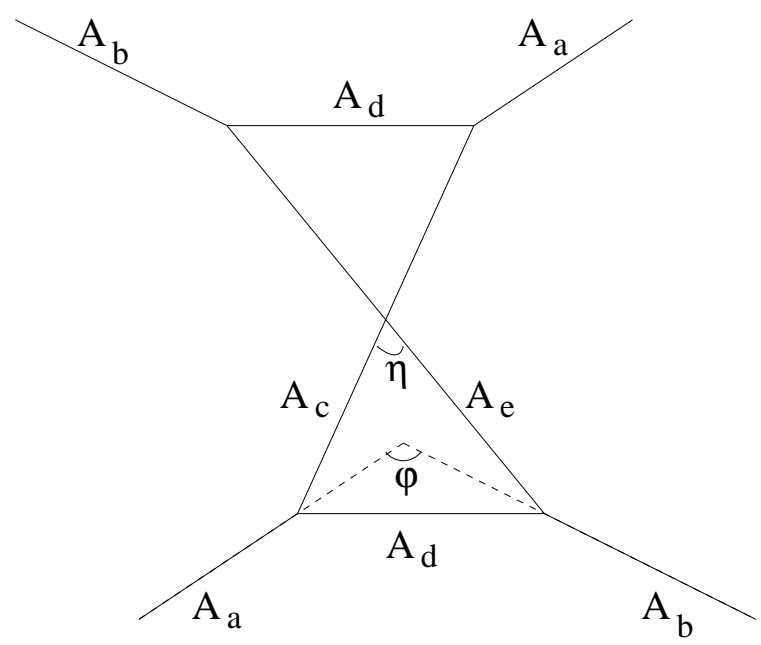

(b)

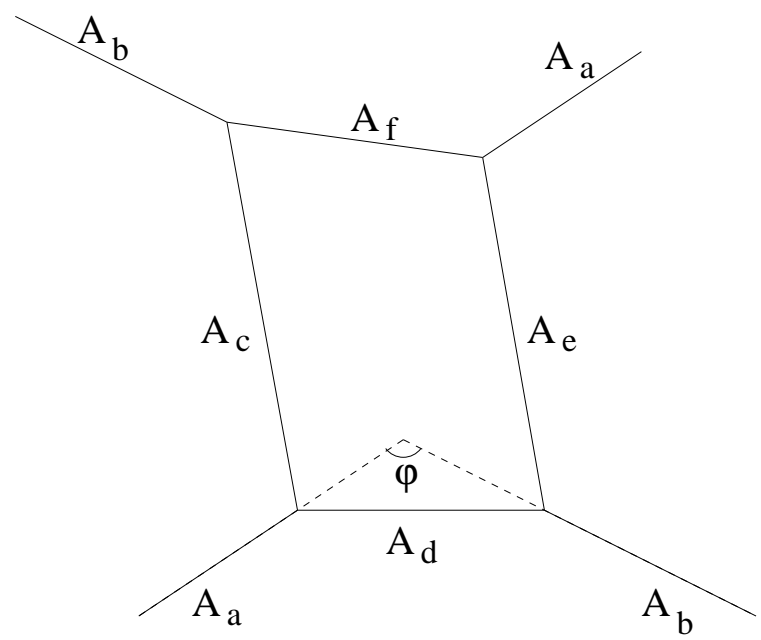

Figure 7: Scattering patterns associated to the second order poles of the $S$-matrix.

for the diagrams of Figures $7 \mathrm{a}$ and $7 \mathrm{~b}$, respectively, and

$$
S_{a b}(\theta \simeq i \varphi) \simeq i \frac{\left(\Gamma_{c d}^{a} \Gamma_{d e}^{b} \Gamma_{e c}^{f}\right)^{2}}{(\theta-i \varphi)^{3}}
$$

for the direct channel third order poles of Figure 8. More generally, a pole of order $P-2 L$ corresponds to a diagram with $P$ internal lines and $L$ loops 40].

We said that the $S$-matrix of Table 1 is the minimal solution to the constraints discussed so far. Although it is extremely natural to expect that this minimal $S$-matrix is the one describing the magnetic Ising model at $T=T_{c}$, the conjecture needs to be checked. Al. Zamolodchikov showed that the conformal limit of an integrable field theory can be identified using the $S$-matrix as the only imput. The method, known as thermodynamic Bethe ansatz, allows the computation of the central charge of the conformal limit through the study of the thermodynamics on a cylindrical geometry 42]. When applied to the $S$-matrix of Table 1 it yields the expected result $C=1 / 2$ [43]. 


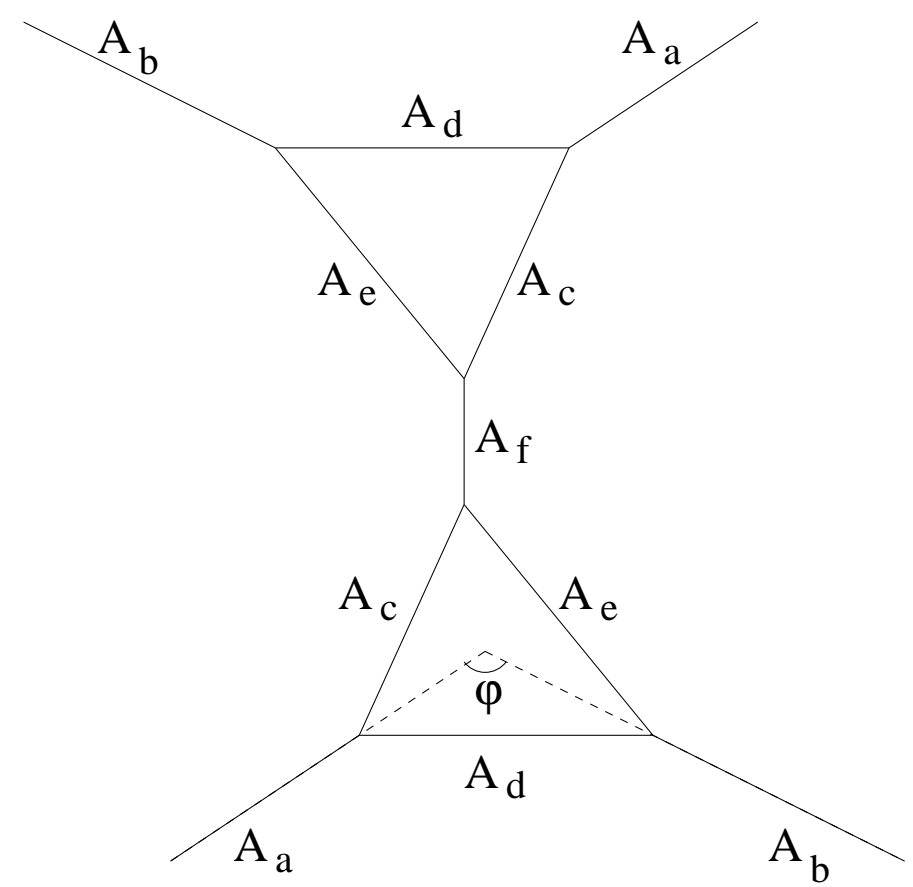

Figure 8: Third order pole of the scattering amplitudes.

A different way of confirming that the $S$-matrix of Table 1 does correspond to the Ising model is that of showing that it describes an integrable quantum field theory containing two relevant scalar operators besides the identity ${ }^{13}$. Addressing this problem means taking Zamolodchikov's $S$-matrix as the imput of the form factor equations (3.25] 3.29) and looking for scalar solutions which behave asymptotically as (3.33) with $Y_{\Phi}<1$ [9, 10]. The non-degenerate spectrum and the " $\varphi^{3}$-property" $\Gamma_{11}^{1} \neq 0$ show that the theory possesses no internal symmetries, so that all the form factors of scalar primaries other than the identity are expected to be non-vanishing. For operators local with respect to the particles $\left(l_{\Phi, \phi_{a}}=1\right)$, the general solution of equations (3.26) and (3.28) with the required pole structure can be written in the form

$$
F_{a_{1} \ldots a_{n}}^{\Phi}\left(\theta_{1}, \ldots, \theta_{n}\right)=Q_{a_{1} \ldots a_{n}}^{\Phi}\left(\theta_{1}, \ldots, \theta_{n}\right) \prod_{i<j} \frac{F_{a_{i} a_{j}}^{\min }\left(\theta_{i}-\theta_{j}\right)}{\left(\cosh \left(\frac{\theta_{i}-\theta_{j}}{2}\right)\right)^{\delta_{a_{i} a_{j}}} D_{a_{i} a_{j}}\left(\theta_{i}-\theta_{j}\right)} .
$$

Here $F_{a b}^{\min }(\theta)$ is a solution of the equations

$$
\begin{aligned}
& F(\theta)=S_{a b}(\theta) F(-\theta) \\
& F(\theta+2 i \pi)=F(-\theta)
\end{aligned}
$$

free of zeroes and poles for $\operatorname{Im} \theta \in(0,2 \pi)$. In the denominator, the factors $\cosh \left(\frac{\theta_{i}-\theta_{j}}{2}\right)$ introduce the annihilation poles, while $D_{a b}(\theta)$ takes care of the dynamical poles in the $A_{a} A_{b}$ scattering channel through factors of the type $\left(\cosh \theta-\cos u_{a b}^{c}\right.$ ) (see later for the precise form). Finally, the $Q_{a_{1} \ldots a_{n}}^{\Phi}$ are entire functions of the rapidities, invariant under exchanges $\theta_{i} \leftrightarrow \theta_{j}$ and (up to a

\footnotetext{
${ }^{13}$ This requirement uniquely identifies the Ising model among the theories satisfying reflection positivity.
} 
sign) $2 \pi i$-periodic in all $\theta_{j}$ 's. They are subject to (3.25) and to the residue equations (3.27) and (3.29) which relate functions with different $n$. These equations, however, hold for any operator with a given spin, so that further constraints are needed to select specific solutions. We will see in a moment the role played in this respect by the asymptotic bound (3.34).

The form factors are determined starting with the first non-trivial case (the two-particle one) and then using the residue equations to fix the matrix elements with an higher number of particles (form factor bootstrap). The specialisation of (4.32) to $n=2$ and scalar operators reads

$$
F_{a b}^{\Phi}(\theta)=\frac{Q_{a b}^{\Phi}(\theta)}{D_{a b}(\theta)} F_{a b}^{\min }(\theta)
$$

where we made the identifications $F_{a b}^{\Phi}\left(\theta_{1}, \theta_{2}\right) \equiv F_{a b}^{\Phi}\left(\theta_{1}-\theta_{2}\right), Q_{a b}^{\Phi}\left(\theta_{1}, \theta_{2}\right) \equiv Q_{a b}^{\Phi}\left(\theta_{1}-\theta_{2}\right)$, and took into account the vanishing residue on the annihilation pole in the two-particle case when $l_{\Phi, \phi_{a}}=1$.

The functions $F_{a b}^{\min }(\theta)$ with the properties specified above can be written as

$$
F_{a b}^{\min }(\theta)=\left(-i \sinh \frac{\theta}{2}\right)^{\delta_{a b}} \prod_{\gamma \in \mathcal{G}_{a b}}\left(T_{\gamma / 30}(\theta)\right)^{p_{\gamma}},
$$

where

$$
T_{\alpha}(\theta)=\exp \left\{2 \int_{0}^{\infty} \frac{d t}{t} \frac{\cosh \left(\alpha-\frac{1}{2}\right) t}{\cosh \frac{t}{2} \sinh t} \sin ^{2} \frac{(i \pi-\theta) t}{2 \pi}\right\}
$$

solves the equations

$$
\begin{gathered}
T_{\alpha}(\theta)=-t_{\alpha}(\theta) T_{\alpha}(-\theta) \\
T_{\alpha}(\theta+2 i \pi)=T_{\alpha}(-\theta)
\end{gathered}
$$

the property

$$
S_{a b}(0)=(-1)^{\delta_{a b}}
$$

of the $S$-matrix originates the factor in front of the product in (4.36).

As we said, the denominator of (4.35) takes care of the dynamical poles. While equation (3.27) says how to deal with the simple poles of the $S$-matrix, the case of higher order poles needs to be discussed [9]. Due to crossing symmetry, poles appear in pairs in the scattering amplitudes. For poles of even order both residues are positive and there is no way to distinguish between a direct and a crossed channel. Then a diagram of the type shown in Figure 7 must exist for each double pole at $\theta=i \varphi$ in the amplitude $S_{a b}(\theta)$. In the vicinity of such a pole, the form factor behaves as ${ }^{14}$ (see Figure 9)

$$
F_{a b}^{\Phi}(\theta \simeq i \varphi) \simeq i \frac{\Gamma_{c d}^{a} \Gamma_{d e}^{b} S_{c e}(i \eta) F_{c e}^{\Phi}(-i \eta)}{\theta-i \varphi}=i \frac{\Gamma_{c d}^{a} \Gamma_{d e}^{b} F_{c e}^{\Phi}(i \eta)}{\theta-i \varphi}
$$

(this result also holds for $\eta=0$ ).

\footnotetext{
${ }^{14}$ The pole in the form factor is simple because the diagram of Figure 9 has a single triangular loop.
} 


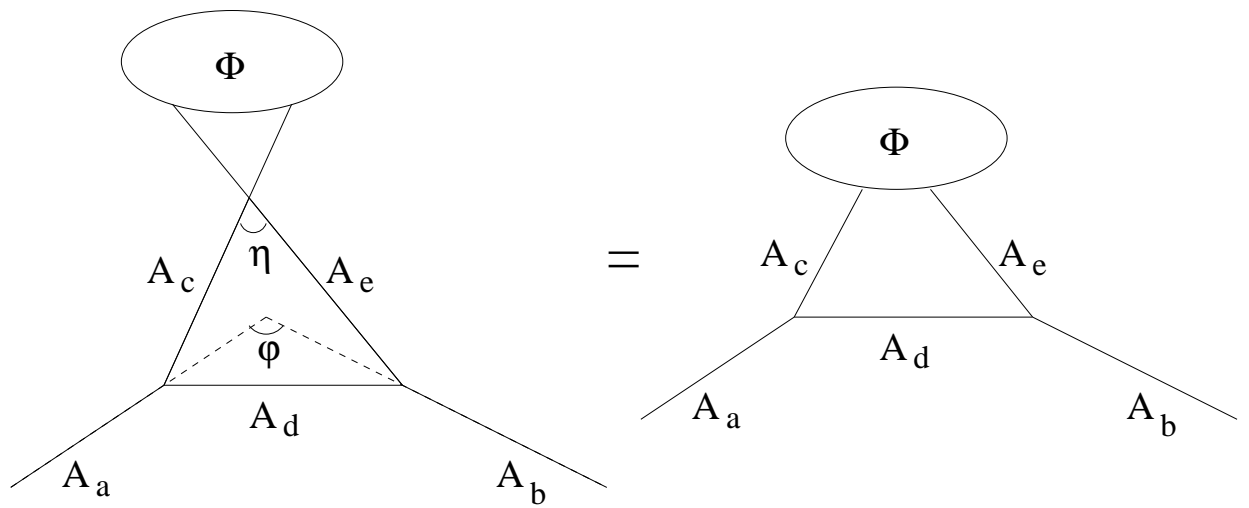

Figure 9: Form factor singularity associated to a double pole of the $S$-matrix.

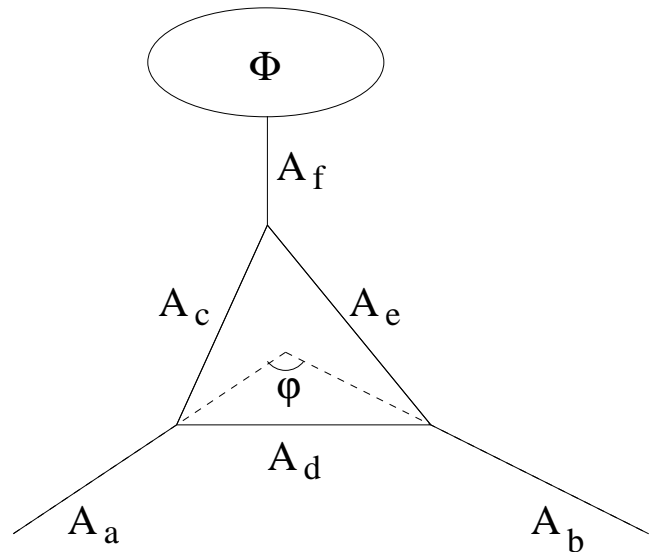

Figure 10: Form factor singularity associated to a direct channel triple pole of the $S$-matrix.

A third order pole in the scattering amplitude can be seen as originating from a double pole when $\eta=u_{c e}^{f}$. Then the direct channel singularity at $\theta=i \varphi$ in the form factor is obtained using (3.27) into (4.41) and reads (Figure 10)

$$
F_{a b}^{\Phi}(\theta \simeq i \varphi) \simeq-\frac{\Gamma_{c d}^{a} \Gamma_{d e}^{b} \Gamma_{e c}^{f}}{(\theta-i \varphi)^{2}} F_{f}^{\Phi}
$$

while the crossed channel pole at $\theta=i(\pi-\varphi)$ remains simple.

The analysis of poles of higher order can be done along similar lines and leads to the expression

$$
D_{a b}(\theta)=\prod_{\gamma \in \mathcal{G}_{a b}}\left(\mathcal{P}_{\gamma / 30}(\theta)\right)^{i_{\gamma}}\left(\mathcal{P}_{1-\gamma / 30}(\theta)\right)^{j_{\gamma}}
$$

where

$$
\mathcal{P}_{\alpha}(\theta)=\frac{\cos \pi \alpha-\cosh \theta}{2 \cos ^{2} \frac{\pi \alpha}{2}}
$$

and

$$
\begin{array}{lll}
i_{\gamma}=n+1, & j_{\gamma}=n, & \text { if } \quad p_{\gamma}=2 n+1 \\
i_{\gamma}=n, & j_{\gamma}=n, & \text { if } \quad p_{\gamma}=2 n .
\end{array}
$$


We are now in the position of dealing with the functions $Q_{a b}^{\Phi}(\theta)$, the only piece of (4.35) which carries information about the operators. As functions of the rapidity difference, they must be even, $2 \pi i$-periodic, free of poles and exponentially bounded. Hence we can write them as

$$
Q_{a b}^{\Phi}(\theta)=\sum_{k=0}^{N_{a b}^{\Phi}} c_{a b, \Phi}^{(k)} \cosh ^{k} \theta
$$

The condition

$$
\left[F_{a b}^{\Phi}(\theta)\right]^{*}=F_{a b}^{\Phi}(-\theta)
$$

follows from (3.26) and $S_{a b}^{*}(\theta)=S_{a b}(-\theta)$, and ensures that the coefficients $c_{a b, \Phi}^{(k)}$ are real. These coefficients are the only unknowns we are left with.

Let us look for solutions corresponding to relevant operators. This means that the asymptotic behaviour (3.33) has to hold with $Y_{\Phi}<1$. Since

$$
T_{\alpha}(\theta) \sim \exp (|\theta| / 2), \quad|\theta| \rightarrow \infty,
$$

it is straightforward to check on (4.35) that, in particular,

$$
N_{11}^{\Phi} \leq 1
$$

Hence, the initial condition of the form factor bootstrap for relevant scalar operators allows for two free parameters, i.e. the coefficients $c_{11, \Phi}^{(0)}$ and $c_{11, \Phi}^{(1)}$. It can be checked that the number of free parameters does not increase when implementing the bootstrap. For example, since

$$
N_{12}^{\Phi} \leq 2
$$

considering $F_{12}^{\Phi}(\theta)$ brings in three more coefficients. On the other hand, the amplitudes $S_{11}(\theta)$ and $S_{12}(\theta)$ possess three common bound states, what yields the three equations

$$
\frac{1}{\Gamma_{11}^{c}} \operatorname{Res}_{\theta=i u_{11}^{c}} F_{11}^{\Phi}(\theta)=\frac{1}{\Gamma_{12}^{c}} \operatorname{Res}_{\theta=i u_{12}^{c}} F_{12}^{\Phi}(\theta), \quad c=1,2,3
$$

which determine the three $c_{12, \Phi}^{(k)}$ in terms of the two $c_{11, \Phi}^{(k)}$. Going on and using also the conditions on higher order poles, a number of residue equations larger than the number of new coefficients is available in many cases. It turns out, however, that the extra constraints are automatically fulfilled so that the two initial parameters propagate untouched through the bootstrap procedure [9] 10].

Since all the form factor equations used so far are linear in the operator $\Phi$, the interpretation of this result is simple: the Zamolodchikov's $S$-matrix describes an integrable field theory with two scaling relevant scalar operators $\Phi_{1}$ and $\Phi_{2}$ (plus the identity) and, up to an additive constant, the operators $\Phi$ selected up to now can be written as

$$
\Phi=\alpha \Phi_{1}+\beta \Phi_{2}
$$

with $\alpha$ and $\beta$ real parameters. We already said that this condition uniquely identifies the Ising field theory (2.12) in which the two scaling operators are $\sigma$ and $\varepsilon$; the known results about the 
integrable directions imply $\tau=0, h \neq 0$. Hence, the analysis of the operator content confirms that the Zamolodchikov's $S$-matrix corresponds to the integrable direction of the magnetic Ising model.

Obviously, the next issue is that of identifying the solutions corresponding to the scaling operators $\sigma$ and $\varepsilon$. Having already exploited all the constraints on form factors discussed so far, it is clear that this task requires some new physical input. Since the scaling operators are characterised by their behaviour close to criticality, it is natural to look once again at the asymptotic properties of form factors. More precisely, we consider the limit 29]

$$
\lim _{\alpha \rightarrow+\infty} F_{a_{1} \ldots a_{r} b_{1} \ldots b_{l}}^{\hat{\Phi}_{k}}\left(\theta_{1}+\frac{\alpha}{2}, \ldots, \theta_{r}+\frac{\alpha}{2}, \theta_{1}^{\prime}-\frac{\alpha}{2}, \ldots, \theta_{l}^{\prime}-\frac{\alpha}{2}\right),
$$

where

$$
\hat{\Phi}_{k}=\frac{\Phi_{k}}{\left\langle\Phi_{k}\right\rangle}, \quad k=1,2
$$

are the scaling operators entering (4.52) rescaled by their vacuum expectation value. It follows from (3.10) that shifting a rapidity $\theta$ by $\pm \alpha / 2$ and rescaling the mass as $m_{a}=M_{a} e^{-\alpha / 2}$ produces, when $\alpha \rightarrow+\infty$, a massless particle with energy $p^{0}= \pm p^{1}=\frac{M_{a}}{2} e^{ \pm \theta}$ (right- or left-mover, depending on the sign of momentum). Hence, (4.53) corresponds to the massless limit towards the conformal point in which the first $r$ particles become right-movers and the remaining $l$ particles left-movers. The property

$$
\lim _{\alpha \rightarrow+\infty} S_{a b}(\theta+\alpha)=1
$$

of the scattering amplitudes of Table 1 shows that a right-mover and a left-mover do not interact in such a conformal limit. We conclude that the limit (4.53) produces a factorisation into two massless form factors, one with $r$ right-movers and one with $l$ left-movers. On the other hand, a massless form factor with all right (left) movers is obtained from (4.53) with $l=0(r=0)$; in such a case all rapidities are shifted by the same amount, and (3.25) shows that this massless form factor actually coincides with the massive one. Then we conclude that the asymptotic factorisation property

$$
\lim _{\alpha \rightarrow+\infty} F_{a_{1} \ldots a_{r} b_{1} \ldots b_{l}}^{\hat{\Phi}_{k}}\left(\theta_{1}+\alpha, \ldots, \theta_{r}+\alpha, \theta_{1}^{\prime}, \ldots, \theta_{l}^{\prime}\right)=F_{a_{1} \ldots a_{r}}^{\hat{\Phi}_{k}}\left(\theta_{1}, \ldots, \theta_{r}\right) F_{b_{1} \ldots b_{l}}^{\hat{\Phi}_{k}}\left(\theta_{1}^{\prime}, \ldots, \theta_{l}^{\prime}\right)
$$

holds in the original massive theory. Notice that the particular cases $r=0$ and/or $l=0$ require the normalisation (4.54). The factorisation argument leading to (4.56) applies to scaling operators and not to linear combinations like (4.52) mixing operators with different scaling dimensions: in the latter case one of the coefficient $\alpha, \beta$ is dimensionful and vanishes in the massless limit.

We are now in the position of identifying the initial conditions of the form factor bootstrap corresponding to the scaling operators $\Phi_{1}$ and $\Phi_{2}$. This amounts to finding the values of the ratio

$$
z_{\Phi}=\frac{c_{11, \Phi}^{(0)}}{c_{11, \Phi}^{(1)}}
$$


for which the factorisation conditions (4.56) are fulfilled (this quantity is universal because does not depend on the normalisation of the operator). The equation

$$
\frac{1}{F_{1}^{\Phi_{k}}} \lim _{\theta \rightarrow \infty} F_{11}^{\Phi_{k}}(\theta)=\frac{1}{F_{2}^{\Phi_{k}}} \lim _{\theta \rightarrow \infty} F_{12}^{\Phi_{k}}(\theta)
$$

is a consequence of (4.56) and gives a quadratic equation for $z_{\Phi}$ whose solutions are [10]

$$
\begin{aligned}
& z_{\Phi_{1}}=4.869840 . . \\
& z_{\Phi_{2}}=1.255585 \ldots
\end{aligned}
$$

Being the operator which perturbs the conformal point, $\sigma$ is proportional to the trace of the energy-momentum tensor. It is not difficult to see that the conservation of the latter induces the factorisation of a kinematical term in the form factors of $\sigma$. In particular, the two-particle form factors $F_{a b}^{\sigma}(\theta)$ contain the factor 9 ]

$$
\left(\cosh \theta+\frac{m_{a}^{2}+m_{b}^{2}}{2 m_{a} m_{b}}\right)^{1-\delta_{a b}} .
$$

It can be checked that the form factors originating from the initial condition $z_{\Phi_{1}}$ have this property. Then we conclude

$$
\begin{aligned}
& \Phi_{1}=\sigma \\
& \Phi_{2}=\varepsilon .
\end{aligned}
$$

Having determined the initial conditions of the form factor bootstrap for the two relevant operators, all their form factors can in principle be computed using the residue equations on dynamical and kinematical poles. The results for several two-particle form factors are given in Table 2; Table 3 contains the full list of one-particle matrix elements [9, 10. Referring to the normalisation-independent ratios (4.54), all these numbers are universal. One can check that the factorisation conditions prescribed by (4.56) are satisfied. Of course, the bootstrap can be continued to determine the remaining two-particle form factors as well as those with more than two particles (for example, $F_{111}^{\sigma}\left(\theta_{1}, \theta_{2}, \theta_{3}\right)$ is computed in [9]).

The content of Tables 2 and 3 is sufficient to develop the large distance expansion (3.22) of two-point correlators including all terms of order lower than $e^{-3 m_{1}|x|}$. In particular, the first few terms are

$$
\left\langle\hat{\Phi}_{k}(x) \hat{\Phi}_{j}(0)\right\rangle=1+\frac{1}{\pi} \sum_{a=1}^{3} F_{a}^{\hat{\Phi}_{k}} F_{a}^{\hat{\Phi}_{j}} K_{0}\left(m_{a}|x|\right)+O\left(e^{-2 m_{1}|x|}\right),
$$

where $K_{0}(z)$ is a Bessel function.

The sum rules (3.35) and (3.36) can be used to test the convergence of the form factor expansion. We recall that $\Theta \sim \sigma$ and that the normalisation of the form factors of $\Theta$ is fixed by the condition (4.11). The asymptotics

$$
\langle\sigma(x) \sigma(0)\rangle \simeq \frac{C_{\sigma \sigma}^{I}}{|x|^{1 / 4}}
$$




\begin{tabular}{|l|r|r|}
\hline & $\hat{\sigma}$ & \multicolumn{1}{|c|}{$\hat{\varepsilon}$} \\
\hline \hline$c_{11}^{1}$ & -2.093102832 & -70.00917205 \\
$c_{11}^{0}$ & -10.19307727 & -87.90247670 \\
\hline$c_{12}^{2}$ & -7.979022182 & -466.3008246 \\
$c_{12}^{1}$ & -71.79206351 & -1307.331521 \\
$c_{12}^{0}$ & -70.29218939 & -853.2803886 \\
\hline$c_{13}^{3}$ & -582.2557366 & -43021.45153 \\
$c_{13}^{2}$ & -6944.416956 & -182413.2733 \\
$c_{13}^{1}$ & -13406.48877 & -241929.7678 \\
$c_{13}^{0}$ & -7049.622303 & -102574.1349 \\
\hline$c_{22}^{3}$ & -21.48559881 & -2193.896354 \\
$c_{22}^{2}$ & -333.8125724 & -10870.05277 \\
$c_{22}^{1}$ & -791.3745549 & -16161.44508 \\
$c_{22}^{0}$ & -500.2535896 & -7510.235388 \\
\hline$c_{14}^{3}$ & 22.57778351 & 2074.636471 \\
$c_{14}^{2}$ & 318.7122159 & 9881.413381 \\
$c_{14}^{1}$ & 672.2210098 & 14357.04570 \\
$c_{14}^{0}$ & 377.4586311 & 6568.762583 \\
\hline$c_{15}^{4}$ & -260.7643072 & -30333.56619 \\
$c_{15}^{3}$ & -4719.877128 & -198757.2340 \\
$c_{15}^{2}$ & -15172.07643 & -447504.5720 \\
$c_{15}^{1}$ & -17428.22924 & -422808.9295 \\
$c_{15}^{0}$ & -6716.787925 & -143743.2050 \\
\hline$c_{23}^{4}$ & -92.73452350 & -11971.94909 \\
$c_{23}^{3}$ & -1846.579035 & -81253.72269 \\
$c_{23}^{2}$ & -6618.297073 & -186593.8661 \\
$c_{23}^{1}$ & -8436.850082 & -178494.3378 \\
$c_{23}^{0}$ & -3579.556465 & -61194.62416 \\
\hline$c_{33}^{5}$ & -1197.056497 & -195385.7662 \\
$c_{33}^{4}$ & -30166.99117 & -1743171.802 \\
$c_{33}^{3}$ & -150512.4122 & -5603957.324 \\
$c_{33}^{2}$ & -301093.9432 & -8422606.859 \\
$c_{33}^{1}$ & -267341.1276 & -6035102.896 \\
$c_{33}^{0}$ & -87821.70785 & -1668721.004 \\
\hline & & \\
\hline
\end{tabular}




\begin{tabular}{|l|r|r|}
\hline$c_{25}^{6}$ & 1425.995027 & 289831.4882 \\
$c_{25}^{5}$ & 44219.03877 & 3275586.983 \\
$c_{25}^{4}$ & 286184.1535 & 13872077.63 \\
$c_{25}^{3}$ & 788413.2178 & 29236961.96 \\
$c_{25}^{2}$ & 1078996.488 & 32979257.31 \\
$c_{25}^{1}$ & 725356.4417 & 19100224.04 \\
$c_{25}^{0}$ & 191383.5734 & 4471623.121 \\
\hline$c_{17}^{5}$ & 190.8548023 & 30394.23374 \\
$c_{17}^{4}$ & 4633.706068 & 274294.8033 \\
$c_{17}^{3}$ & 21406.72691 & 897781.3229 \\
$c_{17}^{2}$ & 39514.82959 & 1375919.456 \\
$c_{17}^{1}$ & 32456.91939 & 1004969.466 \\
$c_{17}^{0}$ & 9906.265607 & 282938.1974 \\
\hline$c_{44}^{7}$ & -7249.785565 & -1830120.693 \\
$c_{44}^{6}$ & -276406.7236 & -25699492.93 \\
$c_{44}^{5}$ & -2299573.212 & -138411873.8 \\
$c_{44}^{4}$ & -849276.3526 & -384776478.8 \\
$c_{44}^{3}$ & -16615618.39 & -608371427.1 \\
$c_{44}^{2}$ & -17950817.11 & -553818699.0 \\
$c_{44}^{1}$ & -10139089.36 & -270964337.7 \\
$c_{44}^{0}$ & -2341590.241 & -55283137.91 \\
\hline
\end{tabular}

Table 2: Coefficients of some of the polynomials (4.46) for the primary operators $\hat{\sigma}$ and $\hat{\varepsilon}$ (parenthesis on the upper index of the coefficients are omitted; $\hat{\Phi}=\Phi /\langle\Phi\rangle$ ).

\begin{tabular}{|r|r|r|}
\hline & \multicolumn{1}{|c|}{$\hat{\sigma}$} & \multicolumn{1}{c|}{$\hat{\varepsilon}$} \\
\hline \hline$F_{1}$ & $-0.64090211 .$. & $-3.70658437 .$. \\
$F_{2}$ & $0.33867436 .$. & $3.42228876 .$. \\
$F_{3}$ & $-0.18662854 .$. & $-2.38433446 .$. \\
$F_{4}$ & $0.14277176 .$. & $2.26840624 .$. \\
$F_{5}$ & $0.06032607 .$. & $1.21338371 .$. \\
$F_{6}$ & $-0.04338937 .$. & $-0.96176431 .$. \\
$F_{7}$ & $0.01642569 .$. & $0.45230320 .$. \\
$F_{8}$ & $-0.00303607 .$. & $-0.10584899 .$. \\
\hline
\end{tabular}

Table 3: The one-particle form factors of the primary operators $\hat{\sigma}$ and $\hat{\varepsilon}(\hat{\Phi}=\Phi /\langle\Phi\rangle)$. 


\begin{tabular}{|l|l|}
\hline$C_{1}$ & 0.472038282 \\
$C_{2}$ & 0.019231268 \\
$C_{3}$ & 0.002557246 \\
$C_{11}$ & 0.003919717 \\
$C_{4}$ & 0.000700348 \\
$C_{12}$ & 0.000974265 \\
$C_{5}$ & 0.000054754 \\
$C_{13}$ & 0.000154186 \\
\hline$C_{\text {partial }}$ & 0.499630066 \\
\hline
\end{tabular}

Table 4: Central charge from a partial sum of the form factor expansion of the correlation function in the sum rule (3.35). $C_{a b . .}$ denotes the contribution of the state $A_{a} A_{b} .$. The exact result is $C=1 / 2$.

$$
\begin{aligned}
\langle\sigma(x) \varepsilon(0)\rangle & \simeq \frac{C_{\sigma \varepsilon}^{\sigma}}{|x|}\langle\sigma\rangle \\
\langle\varepsilon(x) \varepsilon(0)\rangle & \simeq \frac{C_{\varepsilon \varepsilon}^{I}}{|x|^{2}}
\end{aligned}
$$

as $|x| \rightarrow 0$ follow from (2.4) and (2.7) and are helpful in the interpretation of the results of Table 4 and 5 for the sum rules. The faster convergence of the central charge sum rule is expected since the integration in $|x|^{3} d|x|$ strongly suppresses the contribution of short distances. In the scaling dimension sum rule the suppression is lower by a factor $|x|^{2}$ and more effective for $X_{\sigma}$ than for $X_{\varepsilon}$ since $\langle\sigma \sigma\rangle$ is less singular than $\langle\sigma \varepsilon\rangle$.

It is of obvious interest to compare the results of integrable field theory discussed in this section with the numerical results for the original lattice model (2.1) with $T=T_{c}$. Actually, the first numerical investigation was performed in [4] on the Ising quantum spin chain with the purpose of confirming the Zamolodchikov's mass spectrum, what was done with good precision for the first few masses ${ }^{15}$. Concerning correlation functions, after the early studies of [48, 49, the most accurate Monte Carlo simulations have been performed in 50. In this work the numerical data have been used to test the form factor expansion as well as the corrections to the short distance behaviour computed in [51]; the analysis has confirmed the good convergency properties of both expansions.

Caselle and Hasenbusch used a numerical diagonalisation of the transfer matrix of the lattice model to evaluate the first few one-particle form factors [52. The comparison of their results (Table 6) with those of Table 3 provides a direct check of the form factor computations reviewed in this section. The transfer matrix method has also provided a test of two-particle form factors.

\footnotetext{
${ }^{15}$ The full spectrum has been recovered in [45] from the exact solution of the RSOS model of [8], which is in the same universality class of the magnetic Ising model. The spectrum of the field theory (2.12) with $\tau=0$ was also studied in [4] by numerical diagonalisation of the Hamiltonian on the (suitably truncated) space of states of the conformal theory [47.
} 


\begin{tabular}{|l|c|c|}
\hline & $\sigma$ & $\varepsilon$ \\
\hline$\Delta_{1}$ & 0.0507107 & 0.2932796 \\
$\Delta_{2}$ & 0.0054088 & 0.0546562 \\
$\Delta_{3}$ & 0.0010868 & 0.0138858 \\
$\Delta_{11}$ & 0.0025274 & 0.0425125 \\
$\Delta_{4}$ & 0.0004351 & 0.0069134 \\
$\Delta_{12}$ & 0.0010446 & 0.0245129 \\
$\Delta_{5}$ & 0.0000514 & 0.0010340 \\
$\Delta_{13}$ & 0.0002283 & 0.0065067 \\
\hline$\Delta_{\text {partial }}$ & 0.0614934 & 0.4433015 \\
\hline
\end{tabular}

Table 5: Conformal dimensions $\Delta_{\Phi}=X_{\Phi} / 2$ from a partial sum of the form factor expansion of the correlation functions in the sum rule (3.36). $\Delta_{a b .}$ denotes the contribution of the state $A_{a} A_{b} .$. The exact results are $\Delta_{\sigma}=1 / 16=0.0625$ and $\Delta_{\varepsilon}=1 / 2$.

\begin{tabular}{|c|c|l|}
\hline & \multicolumn{1}{|c|}{$\hat{\sigma}$} & \multicolumn{1}{c|}{$\hat{\varepsilon}$} \\
\hline \hline$\left|F_{1}\right|$ & $0.6408(3)$ & $3.707(7)$ \\
$\left|F_{2}\right|$ & $0.325(25)$ & $3.38(7)$ \\
\hline
\end{tabular}

Table 6: Lattice estimates of one-particle form factors obtained in [52].

Indeed, one-point functions on a cylinder of circumference $R$ behave as

$$
\frac{\langle\Phi\rangle_{R}}{\langle\Phi\rangle_{R=\infty}}=1+\frac{1}{\pi} \sum_{a} A_{a}^{\Phi} K_{0}\left(m_{a} R\right)+O\left(e^{-2 m_{1} R}\right), \quad R \rightarrow \infty
$$

where the amplitudes

$$
A_{a}^{\Phi}=\left.\frac{F_{a a}^{\Phi}(i \pi)}{\langle\Phi\rangle}\right|_{R=\infty}
$$

are given in Table 7. The avalaible numerical estimates are [53]

$$
A_{1}^{\sigma}=-8.11(2), \quad A_{1}^{\varepsilon}=-17.5(5) .
$$

\begin{tabular}{|c|c|c|}
\hline & $\sigma$ & $\varepsilon$ \\
\hline \hline$A_{1}$ & $-8.0999744 .$. & $-17.893304 .$. \\
$A_{2}$ & $-21.206008 .$. & $-24.946727 .$. \\
$A_{3}$ & $-32.045891 .$. & $-53.679951 .$. \\
\hline
\end{tabular}

Table 7: Universal amplitudes ruling the leading finite size corrections of one-point functions 54 . 


\section{$5 \quad$ Ising universality class}

Two statistical mechanical systems may differ for a number of microscopic features (e.g the type of lattice or the number of neighbouring sites entering the interaction term) and still be characterised by the same internal symmetry. In this case, the systems will exhibit the same critical behaviour nearby a second order phase transition point associated to the spontaneous breaking of the symmetry. As it is said, they belong to the same universality class. Quantum field theory deals directly with the continuum limit in which the microscopic details become immaterial, and is the natural framework for describing universality classes. In particular, the action (2.12) describes the universality class of the two-dimensional Ising model, and all the results discussed so far with reference to this action are universal. Traditionally, however, the quantitative characterisation of universality in statistical mechanics is made studying the behaviour of the thermodynamical observables in the vicinity of the critical point. In this section we show how integrable field theory allows to complete the list of canonical universal quantities of the Ising universality class [55].

The usual procedure (see [56] and references therein) is that of introducing critical exponents and critical amplitudes through the relations

$$
\begin{aligned}
& \xi= \begin{cases}f_{ \pm}|\tau|^{-\nu}, & \tau \rightarrow 0^{ \pm}, \quad h=0 \\
f_{c}|h|^{-\nu_{c}}, & \tau=0, \quad h \rightarrow 0\end{cases} \\
& C= \begin{cases}\left(A_{ \pm} / \alpha\right)|\tau|^{-\alpha}, & \tau \rightarrow 0^{ \pm}, h=0 \\
\left(A_{c} / \alpha_{c}\right)|h|^{-\alpha_{c}}, & \tau=0, \quad h \rightarrow 0\end{cases} \\
& |\mathcal{M}|=\left\{\begin{array}{lc}
B(-\tau)^{\beta}, & \tau \rightarrow 0^{-}, h=0 \\
(|h| / D)^{1 / \delta}, & \tau=0, \quad h \rightarrow 0
\end{array}\right. \\
& \chi= \begin{cases}\Gamma_{ \pm}|\tau|^{-\gamma}, & \tau \rightarrow 0^{ \pm}, h=0 \\
\Gamma_{c}|h|^{-\gamma_{c}}, & \tau=0, \quad h \rightarrow 0\end{cases}
\end{aligned}
$$

where $\xi$ is the correlation length, $C$ the specific heat, $\mathcal{M}$ the magnetisation and $\chi$ the susceptibility; $\tau$ and $h$ are the deviation from critical temperature and the magnetic field entering the action (2.12). The observables above are related to the one- and two-point functions of the scaling operators $\sigma$ and $\varepsilon$. After introducing the free energy per unit area

$$
f=-\frac{1}{A} \ln Z,
$$


we have ${ }^{16}$

$$
\begin{aligned}
C & =-\frac{\partial^{2} f}{\partial \tau^{2}}=\int d^{2} x\langle\varepsilon(x) \varepsilon(0)\rangle_{c} \\
\mathcal{M} & =-\frac{\partial f}{\partial h}=\langle\sigma\rangle \\
\chi & =-\frac{\partial^{2} f}{\partial h^{2}}=\int d^{2} x\langle\sigma(x) \sigma(0)\rangle_{c} .
\end{aligned}
$$

As for the correlation length, it is common to distinguish between the 'true' correlation length $\xi_{t}$ defined as

$$
\lim _{|x| \rightarrow \infty}\langle\sigma(x) \sigma(0)\rangle_{c} \sim e^{-|x| / \xi_{t}}
$$

and the second moment correlation length

$$
\xi_{2 n d}=\left(\frac{1}{4 \chi} \int d^{2} x|x|^{2}\langle\sigma(x) \sigma(0)\rangle_{c}\right)^{1 / 2}
$$

We will distinguish the critical amplitudes for the two types of correlation length through the superscripts $t$ and $2 n d$.

If $M \sim 1 / \xi$ is a mass scale, we know that $f \sim M^{2}, \tau \sim M^{2-X_{\varepsilon}}$ and $h \sim M^{2-X_{\sigma}}$. Then, comparison of (5.2)-(5.6) with the definitions of the critical exponents gives

$$
\begin{aligned}
& \nu=1 /\left(2-X_{\varepsilon}\right)=1 \\
& \nu_{c}=1 /\left(2-X_{\sigma}\right)=8 / 15 \\
& \alpha=2\left(1-X_{\varepsilon}\right) \nu=0 \\
& \alpha_{c}=2\left(1-X_{\varepsilon}\right) \nu_{c}=0 \\
& \beta=X_{\sigma} \nu=1 / 8 \\
& \delta=1 /\left(X_{\sigma} \nu_{c}\right)=15 \\
& \gamma=2\left(1-X_{\sigma}\right) \nu=7 / 4 \\
& \gamma_{c}=2\left(1-X_{\sigma}\right) \nu_{c}=14 / 15 .
\end{aligned}
$$

These well known results express the fact that the critical exponents are universal and determined by the scaling dimensions of the operators $\sigma$ and $\varepsilon$. They also account for the usual scaling and hyperscaling relations

$$
\begin{aligned}
& \alpha+2 \beta+\gamma=2 \\
& \alpha+2 \nu=2 \\
& \gamma=\beta(\delta-1) \\
& \alpha_{c}=\alpha / \beta \delta \\
& \nu_{c}=\nu / \beta \delta \\
& \gamma_{c}=1-1 / \delta .
\end{aligned}
$$

\footnotetext{
${ }^{16}$ We recall that $\langle\cdots\rangle_{c}$ denotes connected correlators.
} 
Contrary to the exponents, the critical amplitudes are not determined by conformal field theory and are not themselves universal. Roughly speaking, they depend on the scales we use to measure the temperature and the magnetic field (metric factors). It is possible, however, to combine different amplitudes in such a way that the metric factors cancel leaving universal quantities. Such universal amplitude combinations provide the canonical characterisation of the universality of the scaling region surrounding the critical point.

A simple way of canceling the metric factors is to consider ratios like $\Gamma_{+} / \Gamma_{-}, f_{c}^{2 n d} / f_{c}^{t}$ and similar. More sophisticated cancelations are obtained exploiting the relations (5.7)-(5.12) among the critical exponents. So, to (5.7) -(5.11) one associates the universal quantities [56]

$$
\begin{aligned}
& R_{c}=A_{+} \Gamma_{+} / B^{2} \\
& R_{\xi}^{+}=A_{+}^{1 / 2} f_{+}^{t} \\
& R_{\chi}=\Gamma_{+} D B^{\delta-1} \\
& R_{A}=A_{c} D^{-\left(1+\alpha_{c}\right)} B^{-2 / \beta} \\
& Q_{2}=\left(\Gamma_{+} / \Gamma_{c}\right)\left(f_{c}^{t} / f_{+}^{t}\right)^{\gamma / \nu} .
\end{aligned}
$$

Equation (5.12) is instead associated to the identity

$$
\delta \Gamma_{c} D^{1 / \delta}=1
$$

which can be recovered from (3.36) with $\Phi=\sigma$ and

$$
\Theta=-2 \pi h\left(2-X_{\sigma}\right) \sigma, \quad \tau=0 .
$$

Notice that the critical amplitudes of the Ising universality class are defined along the integrable directions of the field theory (2.12). As we are going to see, all of them can be computed exactly. We will work within the so-called 'conformal normalisation' of the operators $\sigma$ and $\varepsilon$, which amounts to take

$$
C_{\sigma \sigma}^{I}=C_{\varepsilon \varepsilon}^{I}=1
$$

in (4.61). We know that the mass $m$ of the particle at $h=0$ and the mass $m_{1}$ of the lightest particle at $\tau=0$ can be written as

$$
\begin{aligned}
& m=\mathcal{C}_{\tau}|\tau| \\
& m_{1}=\mathcal{C}_{h}|h|^{8 / 15} .
\end{aligned}
$$

The two constants are known exactly from a modified version of the thermodynamic Bethe ansatz, and read [57, 58.

$$
\begin{aligned}
& \mathcal{C}_{\tau}=2 \pi \\
& \mathcal{C}_{h}=4.40490857 . .
\end{aligned}
$$

The spontaneous magnetisation at $h=0$ was given in (4.21) and corresponds to the amplitude

$$
B=1.70852190
$$


In the magnetic direction, we can use (4.56) and (5.19) to write

$$
\langle\sigma\rangle=\left[-2 \pi h\left(2-X_{\sigma}\right)\right]^{-1} \frac{\left(F_{1}^{\Theta}\right)^{2}}{\lim _{\theta \rightarrow \infty} F_{11}^{\Theta}(\theta)}, \quad \tau=0
$$

where the form factors of $\Theta$ coincide with those determined in section 4 for $\hat{\sigma}$, up to the normalisation fixed by (4.11). The result for the magnetisation obtained in this way concides with that given by the thermodynamic Bethe ansatz [58], and provides the amplitude

$$
D=0.0253610264 . .
$$

The high- and low-temperature susceptibilities at $h=0$ are obtained integrating the correlators (4.14) and (4.15) with the normalisation (4.21), what gives

$$
\begin{aligned}
& \Gamma_{+}=0.148001214 . . \\
& \Gamma_{-}=0.00392642280 . .
\end{aligned}
$$

The susceptibility amplitude at $\tau=0$ follows from (5.18) and (5.27) and reads

$$
\Gamma_{c}=0.0851721517 .
$$

It appears from (5.5) that the 'true' correlation lenght is just the inverse of the total mass of the lightest particle state entering the form factor expansion of the spin-spin correlator. Then we know from the analysis of section 4 that

$$
\begin{array}{lll}
\xi_{t}= \begin{cases}1 / m, & \tau>0 \\
1 / 2 m, & \tau<0\end{cases} & h=0 \\
\xi_{t}=1 / m_{1}, & \tau=0 .
\end{array}
$$

Concerning the second moment correlation length, at $h=0$ the integration of the exact correlators gives

$$
\xi_{2 n d}=\left\{\begin{array}{ll}
(0.99959808 . .) m, & \tau>0 \\
(0.31607894 . .) m, & \tau<0
\end{array}, \quad h=0 .\right.
$$

At $\tau=0$ we do not know the exact spin-spin correlator, but the integral entering (5.6) is related to the central charge sum rule (3.35) by (5.19). Putting all together one finds the amplitude

$$
f_{c}^{2 n d}=\sqrt{\frac{8}{45 \pi}} D^{1 / 30}=0.21045990 . .
$$

Dealing finally with the specific heat, we have to take into account that, since $\alpha=\alpha_{c}=0$, the critical behaviour gets modified into

$$
C \simeq \begin{cases}-A_{ \pm} \ln |\tau|, & h=0 \\ -A_{c} \ln |h|, & \tau=0 .\end{cases}
$$




\begin{tabular}{c}
$A_{+} / A_{-}=1$ \\
$\Gamma_{+} / \Gamma_{-}=37.6936520 .$. \\
$f_{+}^{t} / f_{-}^{t}=2$ \\
$f_{+}^{2 n d} / f_{+}^{t}=0.99959808 .$. \\
$f_{+}^{2 n d} / f_{-}^{2 n d}=3.16249504 .$. \\
$R_{C}=0.318569391 .$. \\
$R_{\xi}^{+}=1 / \sqrt{2 \pi}=0.39894228 .$. \\
$f_{c}^{2 n d} / f_{c}^{t}=0.9270566 .$. \\
$R_{\chi}=6.77828502 .$. \\
$R_{A}=0.0250658794 .$. \\
$Q_{2}=3.23513834 .$. \\
\hline
\end{tabular}

Table 8: The universal amplitude combinations of the Ising universality class.

On the other hand, (4.61) and (5.20) allow to isolate the logarithmic singularity as

$$
C=\int d^{2} x\langle\varepsilon(x) \varepsilon(0)\rangle_{c} \sim 2 \pi \int_{r_{0}} \frac{d r}{r} \sim-2 \pi \ln \left(M r_{0}\right),
$$

where $r=|x|, r_{0}$ is a short distance cut-off and $M$ is a mass scale needed to make dimensionless the argument of the logarithm. It will be propotional to $m$ at $h=0$ and to $m_{1}$ at $\tau=0$. Recalling (5.21) and (5.22) we conclude

$$
\begin{aligned}
& A_{ \pm}=2 \pi \\
& A_{c}=\frac{16 \pi}{15} .
\end{aligned}
$$

The values of the universal amplitude combinations which follow from these results are collected in Table 8. The first seven quantities involve only amplitudes computed at $h=0$ and are all known since the work of [5]; the last four numbers involve also amplitudes computed along the magnetic direction and where given in [55]. For the latter, an early lattice estimate coming from series expansions is $R_{\chi} \sim 6.78$ [59]; more recently, transfer matrix techniques have given the results $R_{\chi}=6.7782(8)$ and $Q_{2}=3.233(4)$ [52].

\section{Beyond integrability}

We saw in section 3 how two-dimensional quantum field theories allow for integrable directions in coupling space along which a great deal of exact information can be obtained. A number of consequences of this fact for the scaling Ising model have been discussed in the last two sections. It is quite natural to ask how the exact results for the integrable directions can be exploited for the analytic study of the non-integrable ones [60]. In general, we can decompose the action $\mathcal{A}_{N I}$ of the non-integrable theory into the action $\mathcal{A}_{I}$ describing an integrable direction plus the contributions of the scaling operators responsible for the deviation from integrability. 
Considering, for the sake of simplicity, the subspace spanned by only one of such operators, we write

$$
\mathcal{A}_{N I}=\mathcal{A}_{I}-\lambda \int d^{2} x \Psi(x)
$$

In principle, this theory can be studied by perturbation theory in $\lambda$ : all the corrections can be formally written in terms of the matrix elements of the perturbing operator $\Psi$ between particle states in the unperturbed (integrable) theory; as we know, these matrix elements can be computed exactly.

The first order corrections are particularly simple and normally sufficient to explore small deviations from integrability. For example, the leading corrections to the energy spectrum and the scattering amplitudes for the case of neutral particles read 60]

$$
\begin{aligned}
& \delta \mathcal{E}_{v a c}=-\left.\langle\Psi\rangle\right|_{\lambda=0} \lambda+\mathcal{O}\left(\lambda^{2}\right) \\
& \delta m_{a}^{2}=-\left.2 F_{a a}^{\Psi}(i \pi)\right|_{\lambda=0} \lambda+\mathcal{O}\left(\lambda^{2}\right) \\
& \delta S_{a b}(\theta)=\left.i \frac{F_{a b a b}^{\Psi}(\theta)}{m_{a} m_{b}}\right|_{\lambda=0} \frac{\lambda}{\sinh \theta}+\mathcal{O}\left(\lambda^{2}\right),
\end{aligned}
$$

where $\mathcal{E}_{v a c}$ is the energy density of the vacuum state and ${ }^{17}$

$$
\begin{gathered}
F_{a a}^{\Psi}(i \pi) \equiv\left\langle A_{a}(\theta)|\Psi(0)| A_{a}(\theta)\right\rangle_{c}=F_{a a}^{\Psi}(\theta+i \pi, \theta) \\
F_{a b a b}^{\Psi}\left(\theta_{1}-\theta_{2}\right) \equiv\left\langle A_{a}\left(\theta_{1}\right) A_{b}\left(\theta_{2}\right)|\Psi(0)| A_{a}\left(\theta_{1}\right) A_{b}\left(\theta_{2}\right)\right\rangle_{c}=F_{a b a b}^{\Psi}\left(\theta_{1}+i \pi, \theta_{2}+i \pi, \theta_{1}, \theta_{2}\right) .
\end{gathered}
$$

Going to the Ising field theory (2.12), one possibility is to identify the magnetic direction ${ }^{18}$ $\eta=0$ as the integrable unperturbed theory, and to take $\lambda \Psi=\tau \varepsilon$ as the perturbation. Then, in particular, the ratios

$$
\frac{\delta m_{a}^{2}}{\delta \mathcal{E}_{v a c}}=\left.2 F_{a a}^{\hat{\varepsilon}}(i \pi)\right|_{\eta=0}+\mathcal{O}(\eta), \quad \eta \rightarrow 0
$$

are completely universal and can be checked in any numerical approach giving access to the energy spectrum ${ }^{19}$. Their values for $a=1,2,3,4$ follows from the results of Table 2 , which give

$$
\begin{gathered}
\left.F_{11}^{\hat{\hat{\varepsilon}}}(i \pi)\right|_{\eta=0}=-17.8933 . . \\
\left.F_{22}^{\hat{\hat{\varepsilon}}}(i \pi)\right|_{\eta=0}=-24.9467 . . \\
\left.F_{33}^{\hat{\varepsilon}}(i \pi)\right|_{\eta=0}=-53.6799 . . \\
\left.F_{44}^{\hat{\varepsilon}}(i \pi)\right|_{\eta=0}=-49.3169 . .
\end{gathered}
$$

The first few ratios (6.7) have been checked with good accuracy by numerical diagonalisation of the Hamiltonian of the Ising field theory on a conformal basis of states [60], and in the lattice

\footnotetext{
${ }^{17}$ We denote by $\langle\cdots|\cdots| \cdots\rangle_{c}$ the connected part of a matrix element; $\Psi$ is a scalar operator.

${ }^{18}$ The parameter $\eta$ which labels the renormalisation group trajectories in the plane of Figure 1 was defined in (2.13).

${ }^{19}$ When the system is compactified on a cylinder of circumference $R$, the ground state energy behaves as $\mathcal{E}_{v a c} R$ for $R / \xi \gg 1$.
} 
model by numerical diagonalisation of the transfer matrix [61. The absolute variations of the energy levels can be obtained using the result

$$
\left.\langle\varepsilon\rangle\right|_{\eta=0}=(2.00314 . .)|h|^{8 / 15}
$$

which refers to the conformal normalisation (5.20) of the operators and has been obtained in 62] through quite sophisticated techniques; a previous lattice estimate 63] agrees well with this exact value.

Notice that $\delta m_{a}$ will present an imaginary contribution at order $\tau^{2}$ for $a=4, \ldots, 8$. Indeed, we know that at $\tau=0$ the masses $m_{a}$ with $a>3$ lie above the lowest two-particle threshold $2 m_{1}$. There is nothing wrong with this as long as the theory is integrable and all inelastic processes are forbidden. Moving away from $\tau=0$, however, integrability is lost and nothing can prevent the decay $A_{a} \rightarrow A_{1} A_{1}$ when $a>3$. The imaginary part in the mass is inversely proportional to the lifetime of the unstable particle. For $A_{4}$ it is given by 60 ]

$$
\operatorname{Im} m_{4}^{2} \simeq-\left.\frac{\left|F_{411}^{\varepsilon}\left(i \pi, \theta^{*},-\theta^{*}\right)\right|^{2}}{m_{1} m_{4}}\right|_{\eta=0} \frac{\tau^{2}}{2 \sinh \theta^{*}},
$$

with $\theta^{*}$ determined by energy conservation; heavier particles receive similar contributions by other decay channels. These decay widths, as well as the corrections to the scattering amplitudes, can in principle be computed turning the crank of the form factor bootstrap.

In a similar way, the region close to the thermal axis in Figure 1 can be analysed perturbing the $h=0$ theory with the operator $\Psi=\sigma$. Let us start from the low-temperature phase $(\eta=-\infty)$. We know from (6.3) and (4.10) that in this case the first order correction to the mass of the particle of the unperturbed theory is proportional to $\tanh (i \pi / 2)$, and then is infinite. This result is easily understood once we remember that the particle we are dealing with is the kink interpolating between the two vacua of the spontaneously broken phase at $h=0$. As soon as we switch on the magnetic field, the degeneracy of the vacua is lifted, so that all states carrying a topologic charge acquire an infinite energy and are removed from the theory; the single particle excitations of the perturbed theory are kink-antikink bound states $A_{n}$. Hence, the divergence of the mass correction is the signature of the confinement of the topologic charge and manifests itself whenever the perturbing operator $\Psi$ is mutually non-local with respect to the particles of the unperturbed theory ${ }^{20}$ [60, 64]; indeed, (3.29) shows that this is the condition for having a pole at $\theta=i \pi$ in $F_{a a}^{\Psi}(\theta)$.

The magnetic field induces an energy density difference

$$
2\left|\delta \mathcal{E}_{v a c}\right|=2 v h+\mathcal{O}\left(h^{2}\right)
$$

between the two vacua, $v$ being the spontaneous magnetisation (4.21) of the theory at $\eta=-\infty$ ( $v$ takes the sign of $h$ ). Then, neglecting the relativistic effects, a kink and an antikink separated by a distance $R$ are bounded by the potential $2 v h R$. The associated Schrodinger problem gives

\footnotetext{
${ }^{20}$ We recall that in the present case the kink is created by the disorder operator $\mu$.
} 
for the bound states $A_{n}$ the masses

$$
m_{n} \simeq 2 m+\frac{(2 v h)^{2 / 3} z_{n}}{m^{1 / 3}},
$$

where $m$ is the mass of the kink and $z_{n}, n=1,2, \ldots$, are positive numbers determined by the zeroes of the Airy function, $\operatorname{Ai}\left(-z_{n}\right)=0$. This non-relativistic approximation holds as long as $m_{n}-2 m \ll m$, and then gives the correct leading behaviour for $\eta \rightarrow-\infty$. Of course, the particles with masses $m_{n}$ larger than twice the lightest mass $m_{1}$ are unstable. The masses $m_{n}$ become dense above $2 m$ as $\eta \rightarrow-\infty$, and condensate to reproduce the continuum spectrum when $h$ vanishes. The spectrum (6.15) was first obtained in 65] through the study of the analytic structure in momentum space of the spin-spin correlation function for small magnetic field. Relativistic corrections to (6.15) have recently been obtained in [66, 35].

It is possible at this point to figure out the evolution of the particle spectrum of the Ising field theory in the plane of Figure 1. We have just described how the kinks living at $\eta=$ $-\infty$ are confined into a tower of bound states when the magnetic field is switched on. The number of stable particles (i.e. with $m_{n}<2 m_{1}$ ) decreases as $\eta$ is increased from $-\infty$ to finite negative values. We expect three such particles to be left for small values of $\eta$ (i.e. close to the magnetic axis), although five more particles above threshold happen to be stable at $\eta=0$ due to integrability. The number of stable particles continues to decrease as $\eta$ increases, until a single particle is left at $\eta=+\infty$ (the positive thermal axis). This scenario, which is an updated version of that originally proposed in [65, is supported by the numerical investigation of the spectrum of the Ising field theory [60, 66].

The correction to the mass of the particle when the magnetic field is switched on at $\tau>0$ can again be computed in perturbation theory. This time, however, the first order correction vanishes for symmetry reasons (the perturbing operator $\sigma$ couples only to an odd number of particles at $\eta=+\infty)$, and one has

$$
\delta m_{1}^{2}=b m^{-7 / 4} h^{2}+\mathcal{O}\left(h^{4}\right),
$$

where $m$ is the mass at $\eta=+\infty$, and

$$
b=-m^{7 / 4} \int d^{2} x\langle A(\theta)|\sigma(x) \sigma(0)| A(\theta)\rangle_{c}
$$

(the integral is evaluated at $\eta=+\infty$ ). In principle, one could think to evaluate the matrix element through a decomposition over intermediate particle states. Very recently, however, Fonseca and A. Zamolodchikov computed it generalising the techniques used for the spin-spin correlation function at $h=0$. In this way they found [35]

$$
b=21.52398
$$

within the normalisation $(5.20)$ of the operators. Since

$$
\delta \mathcal{E}_{v a c}=-\frac{h^{2}}{2} \int d^{2} x\langle\sigma(x) \sigma(0)\rangle_{c}+\mathcal{O}\left(h^{4}\right)=-\frac{\Gamma_{+}}{2} \tau^{-7 / 4} h^{2}+\mathcal{O}\left(h^{4}\right),
$$


we have

$$
\frac{\delta m_{1}^{2}}{\delta \mathcal{E}_{\text {vac }}}=-\frac{2 b}{\Gamma_{+}}(2 \pi)^{-7 / 4}+\mathcal{O}\left(\eta^{-15 / 4}\right)=-(11.66467 . .)+\mathcal{O}\left(\eta^{-15 / 4}\right), \quad \eta \rightarrow+\infty .
$$

We mention, in conclusion, that 66] contains also a detailed study, which combines analytic expansions and numerical methods, of the analytic properties of the free energy of the Ising field theory as a function of temperature and magnetic field.

Acknowledgments. I thank J. Cardy, G. Mussardo and P. Simonetti for collaborating with me on several topics reviewed in this article. This work was partially supported by the European Commission TMR programme HPRN-CT-2002-00325 (EUCLID) and by the COFIN "Teoria dei Campi, Meccanica Statistica e Sistemi Elettronici". 


\section{References}

[1] E. Ising, Z. Physik 31 (1925) 253.

[2] L. Onsager, Phys. Rev. 65 (1944) 117.

[3] C.N. Yang, Phys. Rev. 85 (1952) 808.

[4] B.M. McCoy and T.T. Wu, The Two-Dimensional Ising Model, Harvard University Press, Cambridge, Massachussets, 1973.

[5] T.T. Wu, B.M. Mccoy, C.A. Tracy and E. Barouch, Phys. Rev. B 13 (1976) 316.

[6] A.B. Zamolodchikov, Advanced Studies in Pure Mathematics 19 (1989) 641; Int. J. Mod. Phys. A 3 (1988) 743.

[7] A.A. Belavin, A.M. Polyakov and A.B. Zamolodchikov, Nucl. Phys. B 241 (1984) 333.

[8] S.O. Waarnar, B. Nienhuis and K.A. Seaton, Phys. Rev. Lett. 69 (1992) 710.

[9] G. Delfino and G. Mussardo, Nucl. Phys. B 455 (1995) 724.

[10] G. Delfino and P. Simonetti, Phys. Lett. B 383 (1996) 450.

[11] H.A. Kramers and G.H. Wannier, Phys. Rev. 60 (1941) 252 and 263.

[12] J.L. Cardy, Scaling and renormalization in statistical physics, Cambridge University Press, Cambridge, 1996.

[13] D. Friedan, Z. Qiu and S. Shenker, Phys. Rev. Lett. 52 (1984) 1575.

[14] L.P. Kadanoff and H. Ceva, Phys. Rev. B 3 (1971) 3918.

[15] G. Mussardo, Phys. Rep. 218 (1992) 215.

[16] P.D. Fonseca, Mod. Phys. Lett. A 13 (1998) 1931.

[17] A.B. Zamolodchikov and Al.B. Zamolodchikov, Ann. Phys. 120 (1979) 253.

[18] R. Shankar and E. Witten, Phys. Rev. D 17 (1978) 2134.

[19] D. Iagolnitzer, Phys. Lett. B 76 (1978) 207; Phys. Rev. D 18 (1978) 1275.

[20] S. Parke, Nucl. Phys. B 174 (1980) 166.

[21] R.J. Eden, P.V. Landshoff, D.I. Olive and J.C. Polkinghorne, The analytic S-matrix, Cambridge University Press, 1966.

[22] P. Mitra, Phys. Lett. B 72 (1977) 62.

[23] M. Karowski, P. Weisz, Nucl. Phys. B 139 (1978) 455. 
[24] F.A. Smirnov, Form Factors in Completely Integrable Models of Quantum Field Theory, World Scientific, 1992.

[25] V.P. Yurov and Al.B. Zamolodchikov, Int. J. Mod. Phys. A 6 (1991) 3419.

[26] Al.B. Zamolodchikov, Nucl. Phys. B 348 (1991) 619.

[27] A.B. Zamolodchikov, JETP Lett. 43 (1986) 730.

[28] J.L. Cardy, Phys. Rev. Lett. 60 (1988) 2709.

[29] G. Delfino, P. Simonetti and J.L. Cardy, Phys. Lett. B 387 (1996) 327.

[30] B. Berg, M. Karowski, P. Weisz, Phys. Rev. D 19 (1979) 2477.

[31] J.L. Cardy and G. Mussardo, Nucl. Phys. B 340 (1990) 387.

[32] O. Babelon and D. Bernard, Phys. Lett. B 288 (1992) 113.

[33] D. Bernard and A. LeClair, Nucl. Phys. B 426 (1994) 534; Erratum-ibid. B 498 (1997) 619.

[34] M. Sato, T. Miwa and T. Jimbo, Publ. RIMS Kyoto Univ. 14 (1978) 223.

[35] P. Fonseca and A.B. Zamolodchikov, hep-th/0309228.

[36] J. Balog, M. Niedermaier, F. Niedermayer, A. Patrascioiu, E. Seiler and P. Weisz, Nucl. Phys. B 583 (2000) 614.

[37] V.A. Fateev, unpublished.

[38] V.A. Fateev and A.B. Zamolodchikov, Int. J. Mod. Phys. A 5 (1990) 1025.

[39] S. Coleman and H.J. Thun, Commun. Math. Phys. 61 (1978) 31.

[40] H.W. Braden, E. Corrigan, P.E. Dorey and R. Sasaki, Nucl. Phys. B 338 (1990) 689.

[41] P. Christe and G. Mussardo, Nucl. Phys. B 330 (1990) 465.

[42] Al.B. Zamolodchikov, Nucl. Phys. B 342 (1990) 695.

[43] T.R. Klassen and E. Melzer, Nucl. Phys. B 338 (1990) 485.

[44] M. Henkel and H. Saleur, J. Phys. A 22 (1989) L513.

[45] V.V. Bazhanov, B. Nienhuis, and S.O. Warnaar, Phys. Lett. B 322 (1994) 198.

[46] I.R. Sagdeev and A.B. Zamolodchikov, Mod. Phys. Lett. B 3 (1989) 1375.

[47] V.P. Yurov and Al.B. Zamolodchikov, Int. J. Mod. Phys. A 6 (1991) 4557.

[48] P.G. Lauwers and V. Rittenberg, Phys. Lett. B 233 (1989) 197; Bonn preprint HE-89-11. 
[49] C. Destri, F. Di Renzo, E. Onofri, P. Rossi and G.P. Tecchiolli, Phys. Lett. B 278 (1992) 311.

[50] M. Caselle, P. Grinza and N. Magnoli, Nucl. Phys. B 579 (2000) 635.

[51] R. Guida and N. Magnoli, Nucl. Phys. B 483 (1997) 563.

[52] M. Caselle and M. Hasenbusch, Nucl. Phys. B 579 (2000) 667.

[53] M. Caselle and M. Hasenbusch, Nucl. Phys. B 639 (2002) 549.

[54] G. Delfino, J. Phys. A 34 (2001) L161.

[55] G. Delfino, Phys. Lett. B 419 (1998) 291; Erratum-ibid. B 518 (2001) 330.

[56] V. Privman, P.C. Hohenberg and A. Aharony, Universal critical point amplitude relations, in 'Phase transition and critical phenomena', Vol. 14, C. Domb and J.L. Lebowitz eds., Academic Press, 1991.

[57] Al.B. Zamolodchikov, Int. J. Mod. Phys. A 10 (1995) 1125.

[58] V.A. Fateev, Phys. Lett. B 324 (1994) 45.

[59] H.B. Tarko and M.E. Fisher, Phys. Rev. B 11 (1975) 1217.

[60] G. Delfino, G. Mussardo and P. Simonetti, Nucl. Phys. B 473 (1996) 469.

[61] P. Grinza and A. Rago, Nucl. Phys. B 651 (2003) 387.

[62] V.A. Fateev, S. Lukyanov, A.B. Zamolodchikov and Al.B. Zamolodchikov, Nucl. Phys. B 516 (1998) 652 .

[63] R. Guida and N. Magnoli, Phys. Lett. B 411 (1997) 127.

[64] G. Delfino and G. Mussardo, Nucl. Phys. B 516 (1998) 675.

[65] B.M. McCoy and T.T. Wu, Phys. Rev. D 18 (1978) 1259.

[66] A.B. Zamolodchikov and P. Fonseca, J. Stat. Phys. 110 (2003) 527. 